\title{
Long-Lived Fission Product Transmutation Studies
}

\author{
W. S. Yang,* Y. Kim, † R. N. Hill, T. A. Taiwo, and H. S. Khalil \\ Argonne National Laboratory, Nuclear Engineering Division \\ 9700 S. Cass Avenue, Argonne, Illinois 60439
}

Received March 26, 2003

Accepted June 30, 2003

\begin{abstract}
A systematic study on long-lived fission products (LLFPs) transmutation has been performed with the aim of devising an optimal strategy for their transmutation in critical or subcritical reactor systems and evaluating impacts on the geologic repository. First, ${ }^{99} \mathrm{Tc}$ and ${ }^{129}$ I were confirmed to have highest transmutation priorities in terms of transmutability and long-term radiological risk reduction. Then, the transmutation potentials of thermal and fast systems for ${ }^{99} \mathrm{Tc}$ and ${ }^{129} \mathrm{I}$ were evaluated by considering a typical pressurized water reactor $(P W R)$ core and a sodium-cooled accelerator transmutation of waste system. To determine the best transmutation capabilities, various target design and loading optimization studies were performed. It was found that both ${ }^{99}$ Tc and ${ }^{129}$ I can be stabilized (i.e., zero net production) in the same PWR core under current design constraints by mixing ${ }^{99}$ Tc with fuel and by loading $\mathrm{CaI}_{2}$ target pins mixed with $\mathrm{ZrH}_{2}$ in guide tubes, but the PWR option appears to have a limited applicability as a burner of legacy LLFP. In fast systems, loading of moderated LLFP target assemblies in the core periphery (reflector region) was found to be preferable from the viewpoint of neutron economy and safety. By a simultaneous loading of ${ }^{99} \mathrm{Tc}$ and ${ }^{129} \mathrm{I}$ target assemblies in the reflector region, the self-generated ${ }^{99} \mathrm{Tc}$ and ${ }^{129} \mathrm{I}$ as well as the amount produced by several PWR cores could be consumed at a cost of $\sim 10 \%$ increased fuel inventory. Discharge burnups of $\sim 29$ and $\sim 37 \%$ are achieved for ${ }^{99}$ Tc and ${ }^{129}$ I target assemblies with an $\sim 5$-yr irradiation period.

Based on these results, the impacts of ${ }^{99} \mathrm{Tc}$ and ${ }^{129}$ I transmutation on the Yucca mountain repository were assessed in terms of the dose rate. The current Yucca Mountain release evaluations do not indicate a compelling need to transmute ${ }^{99}$ Tc and ${ }^{129}$ I because the resulting dose rates fall well below current regulatory limits. However, elimination of the LLFP inventory could allow significant relaxation of the waste form and container performance criteria, with associated economic benefits. Therefore, some development of either specialized waste form or transmutation target for the LLFP is prudent, especially considering the potential accumulation of large LLFP inventory with sustained use of nuclear energy into the future.
\end{abstract}

\section{INTRODUCTION}

As an approach to minimize the nuclear waste sent to the geologic repository and hence to increase the effective repository capacity, the potential to recycle and incinerate the radiotoxic transuranics (TRU) in nuclear reactor systems has been studied. ${ }^{1,2}$ If the TRU inventory is reduced significantly by deploying TRU transmuters, the long-lived fission products (LLFPs) will likely

*E-mail: wyang@rae.anl.gov

$\dagger$ Permanent address: Korea Atomic Energy Research Institute, Yusong, P.O. Box 105, Daejeon 305-600, Korea. dominate the long-term dose associated with radionuclide release from the geologic repository. To reduce the long-term dose, it has been suggested to transmute LLFPs into stable or short-lived isotopes by neutron capture in reactor systems.

Various studies of the LLFP transmutation in reactor systems have previously been performed, ${ }^{3-10}$ ranging from simple estimations of transmutability based on physical properties of various LLFPs to actual irradiation tests in experimental reactors. However, to the best knowledge of the authors, most of the studies were scoping-type analyses to estimate the potential transmutation rates in specific systems with limited consideration of design and safety constraints. In addition, the overall net benefits of 
the LLFP transmutation relative to the direct geologic disposal have not been investigated systematically.

To devise an optimal strategy for LLFP transmutation in critical or subcritical reactor systems and to evaluate its impacts on the geologic repository, a systematic study on LLFP transmutation has been performed. First, the open literature information was collected and thoroughly assessed, to decide the order of transmutation priorities for various LLFPs. Then, the transmutation potentials of thermal and fast systems for ${ }^{99} \mathrm{Tc}$ and ${ }^{129} \mathrm{I}$ were evaluated for a typical pressurized water reactor (PWR) core and a sodium-cooled accelerator transmutation of waste (ATW) system. ${ }^{11}$ To identify favorable transmutation strategies, various target design and loading optimization studies were performed under various design constraints. The impacts of LLFP loading on safety parameters and core performance parameters were also evaluated. Finally, based on these results, the impacts of ${ }^{99} \mathrm{Tc}$ and ${ }^{129} \mathrm{I}$ transmutation on the Yucca mountain repository ${ }^{12}$ were assessed in terms of the released dose rate.

The organization of this paper is as follows: Section II provides a summary on the general characteristics of important LLFPs and their transmutability in reactor systems. The LLFP transmutation potentials of a typical PWR and a sodium-cooled ATW system are discussed in Secs. III and IV, respectively. In Sec. V, the impact of LLFP transmutation on repository performance is described. The conclusions and future work recommendations are presented in Sec. VI.

\section{TRANSMUTATION CHARACTERISTICS OF LONG-LIVED FISSION PRODUCTS}

Transmutation of toxic LLFPs makes sense only if the total radiotoxicity of nuclides being sent to a geologic repository is reduced significantly. This requires that the target FP should interact with neutrons at a much higher rate than natural decay. In addition, the radiotoxicity of the product isotopes (including the activation products of the materials used in the target fabrication) should be much smaller than the initial radiotoxicity. In order to identify the candidate LLFPs and appropriate target forms, basic property data in the open literature pertaining to LLFP transmutation were collected and assessed.

The radiological properties of major LLFPs and their production rates in a typical PWR core are summarized in Table I. The radiotoxicity values were estimated using the International Commission on Radiation Protection (ICRP) data, and the production rates were calculated for a typical three-batch PWR core using the ORIGEN2 code. ${ }^{13}$ From Table I, it can be seen that ${ }^{90} \mathrm{Sr}$ and ${ }^{137} \mathrm{Cs}$ have a large radiotoxicity due to their relatively short half-life. Except for ${ }^{107} \mathrm{Pd}$, all beta decays accompany
TABLE I

Radiological Properties of Major LLFPs and Production Rates in PWRs

\begin{tabular}{|c|c|c|c|c|}
\hline \multirow[b]{2}{*}{ Isotope } & \multicolumn{2}{|c|}{ Decay } & \multirow{2}{*}{$\begin{array}{c}\text { Production }{ }^{\mathrm{a}} \\
{[\mathrm{kg} / \mathrm{GW}(\text { ther- }} \\
\mathrm{mal}) \cdot \mathrm{yr}]\end{array}$} & \multirow[b]{2}{*}{$\begin{array}{c}\text { Radiotoxicity } \\
\text { (Sv/g) }\end{array}$} \\
\hline & Type & $\begin{array}{l}\text { Half-Life } \\
(y r)\end{array}$ & & \\
\hline${ }^{79} \mathrm{Se}$ & $\beta^{-}$ & $6.5 \mathrm{E}+4^{\mathrm{c}}$ & 0.066 & 8.259 \\
\hline${ }^{90} \mathrm{Sr}$ & $\beta^{-}$ & 29 & 6.07 & $1.269 \mathrm{E}+5$ \\
\hline${ }^{93} \mathrm{Zr}$ & $\beta^{-}$ & $1.5 \mathrm{E}+5$ & 8.04 & 1.045 \\
\hline${ }^{94} \mathrm{Nb}$ & $\beta^{-}$ & $2.0 \mathrm{E}+4$ & $8.1 \mathrm{E}-6$ & $1.410 \mathrm{E}+1$ \\
\hline${ }^{99} \mathrm{Tc}$ & $\beta^{-}$ & $2.1 \mathrm{E}+5$ & 8.54 & $6.056 \mathrm{E}-1$ \\
\hline${ }^{107} \mathrm{Pd}$ & $\beta^{-}$ & $6.5 \mathrm{E}+6$ & 2.34 & $1.048 \mathrm{E}-3$ \\
\hline${ }^{126} \mathrm{Sn}$ & $\beta^{-}$ & $1.0 \mathrm{E}+5$ & 0.30 & 6.306 \\
\hline${ }^{129} \mathrm{I}$ & $\beta^{-}$ & $1.6 \mathrm{E}+7$ & 1.96 & $2.696 \mathrm{E}-1$ \\
\hline${ }^{135} \mathrm{Cs}$ & $\beta^{-}$ & $2.3 \mathrm{E}+6$ & 2.76 & $8.532 \mathrm{E}-2$ \\
\hline${ }^{137} \mathrm{Cs}$ & $\beta^{-}$ & 30 & 10.65 & $4.190 \mathrm{E}+4$ \\
\hline${ }^{151} \mathrm{Sm}$ & $\beta^{-}$ & 89 & 0.15 & $1.281 \mathrm{E}+2$ \\
\hline
\end{tabular}

a3.2\% ${ }^{235} \mathrm{U}$ enrichment, $33 \mathrm{GWd} /$ tonne $\mathrm{U}, 20$-yr cooling.

bICRP data.

${ }^{\mathrm{c}}$ Read as $6.5 \times 10^{4}$.

subsequent gamma decays. Palladium-107 has the smallest radiotoxicity (measured in sievert per gram) since its beta energy is only $30 \mathrm{keV}$.

The transmutation rate of an LLFP in a neutron field can be characterized by the transmutation half-life defined as the time needed to incinerate half of its initial mass by neutron capture. This transmutation half-life can be defined as

$$
T_{1 / 2}^{t r}=3.171 \times 10^{-8} \frac{\ln 2}{\sigma_{\gamma} \Phi} \quad[\mathrm{yr}],
$$

where

$$
\begin{aligned}
\sigma_{\gamma} & =\text { neutron capture cross section (b) } \\
\Phi & =\text { neutron flux }\left(\mathrm{n} / \mathrm{cm}^{2} \cdot \mathrm{s}\right) .
\end{aligned}
$$

For effective LLFP transmutation, the transmutation half-life $T_{1 / 2}^{t r}$ should be much smaller than the natural decay half-life $T_{1 / 2}$. To estimate the generic transmutation potential, transmutation half-lives were calculated for typical fast and thermal systems using the ORIGEN2 code.

Table II compares these transmutation half-lives with the natural decay half-lives. It can be seen that six nuclides $\left({ }^{94} \mathrm{Nb},{ }^{99} \mathrm{Tc},{ }^{107} \mathrm{Pd},{ }^{129} \mathrm{I},{ }^{135} \mathrm{Cs}\right.$, and $\left.{ }^{151} \mathrm{Sm}\right)$ are transmutable in either thermal or fast reactors. Thus, these are possible candidate LLFPs for reactor-based transmutation. Other nuclides are very difficult to transmute in reactor systems because of their very small capture cross section: Extremely high flux level would be required for effective transmutation. Even though capture cross 
TABLE II

Generic Transmutability of LLFPs in Fast and Thermal Neutron Fields

\begin{tabular}{|c|c|c|c|c|c|c|}
\hline \multirow[b]{2}{*}{ Isotope } & \multicolumn{2}{|c|}{ Capture Cross Section ${ }^{a}$} & \multirow[b]{2}{*}{$\begin{array}{l}\text { Half-Life } \\
\text { (yr) }\end{array}$} & \multicolumn{2}{|c|}{ Transmutation Half-Life ${ }^{b}$} & \multirow[b]{2}{*}{$\begin{array}{c}\text { Pure Isotope } \\
\text { Transmutability }\end{array}$} \\
\hline & $\begin{array}{c}\text { Fast } \\
\text { Neutron }\end{array}$ & $\begin{array}{l}\text { Thermal } \\
\text { Neutron }\end{array}$ & & $\begin{array}{c}\text { Fast } \\
\text { Neutron }\end{array}$ & $\begin{array}{l}\text { Thermal } \\
\text { Neutron }\end{array}$ & \\
\hline${ }^{79} \mathrm{Se}$ & 0.002 & 0.33 & $6.5 \mathrm{E}+4^{\mathrm{c}}$ & $1.1 \mathrm{E}+4$ & 666 & Nontransmutable \\
\hline${ }^{90} \mathrm{Sr}$ & 0.01 & 0.08 & 29 & $2.2 \mathrm{E}+3$ & $2.7 \mathrm{E}+3$ & Nontransmutable \\
\hline${ }^{93} \mathrm{Zr}$ & 0.09 & 1.03 & $1.5 \mathrm{E}+5$ & 244 & 213 & Questionable \\
\hline${ }^{94} \mathrm{Nb}$ & 0.22 & 4.22 & $2.0 \mathrm{E}+4$ & 100 & 52 & Transmutable \\
\hline${ }^{99} \mathrm{Tc}$ & 0.45 & 9.32 & $2.1 \mathrm{E}+5$ & 49 & 24 & Transmutable \\
\hline${ }^{107} \mathrm{Pd}$ & 0.53 & 2.79 & $6.5 \mathrm{E}+6$ & 42 & 79 & Transmutable \\
\hline${ }^{126} \mathrm{Sn}$ & 0.007 & 0.03 & $1.0 \mathrm{E}+5$ & $3.1 \mathrm{E}+3$ & $7.3 \mathrm{E}+3$ & Nontransmutable \\
\hline${ }^{129} \mathrm{I}$ & 0.35 & 3.12 & $1.6 \mathrm{E}+7$ & 63 & 70 & Transmutable \\
\hline${ }^{135} \mathrm{Cs}$ & 0.07 & 2.48 & $2.3 \mathrm{E}+6$ & 314 & 89 & Transmutable \\
\hline${ }^{137} \mathrm{Cs}$ & 0.01 & 0.03 & 30 & $2.2 \mathrm{E}+3$ & $7.3 \mathrm{E}+3$ & Nontransmutable \\
\hline${ }^{151} \mathrm{Sm}$ & 2.09 & 660 & 89 & 11 & 0.33 & Transmutable \\
\hline
\end{tabular}

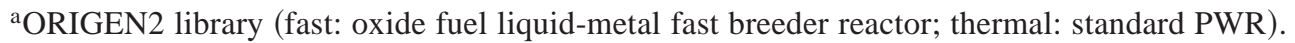

${ }^{\mathrm{b}}$ Thermal flux $=1.0 \times 10^{14}$, fast flux $=1.0 \times 10^{15}\left(\mathrm{n} / \mathrm{cm}^{2} \cdot \mathrm{s}\right)$.

${ }^{\mathrm{c}}$ Read as $6.5 \times 10^{4}$.

sections are higher in thermal systems than in fast systems, thermal systems do not provide a decisive advantage over fast systems because of their lower flux level. However, in a given system, softening of the local energy spectrum usually provides better transmutation efficiency.

It is generally recognized that isotopic separation of LLFPs would be very difficult and costly. Thus, if an LLFP requires isotopic separation for effective transmutation, it cannot be transmuted efficiently from a practical point of view. For the above six transmutable LLFPs, the isotopic compositions and the corresponding capture cross sections for a standard PWR spectrum are summarized in Table III. It can be seen that ${ }^{135} \mathrm{Cs}$ strongly re- quires isotopic separation for effective transmutation because of the much higher weight fractions and capture cross sections of ${ }^{133} \mathrm{Cs}$ and ${ }^{134} \mathrm{Cs}$; in direct transmutation of elemental cesium, additional ${ }^{135} \mathrm{Cs}$ would be produced from ${ }^{133} \mathrm{Cs}$ and ${ }^{134} \mathrm{Cs}$ by neutron capture. In addition, very strong gamma rays emitted by ${ }^{137} \mathrm{Cs}$ would make handling and isotopic separation of cesium very difficult. Niobium-94 and ${ }^{107} \mathrm{Pd}$ also need isotopic separation for efficient transmutation. On the other hand, ${ }^{151} \mathrm{Sm}$ does not require isotope separation due to its very large capture cross section, even though the weight fraction of ${ }^{150} \mathrm{Sm}$ is quite large. For ${ }^{129} \mathrm{I}$, isotopic separation is not strongly required because the ${ }^{127} \mathrm{I}$ fraction is relatively small, and its capture product ${ }^{128}$ I quickly decays

TABLE III

Isotopic Composition of Transmutable LLFPs

\begin{tabular}{|c|c|}
\hline Isotope & $\begin{array}{c}\text { Isotopic Composition } \\
\left(\text { wt } \% ; \text { capture cross section) }{ }^{\mathrm{a}}\right.\end{array}$ \\
\hline $\mathrm{Nb}$ & ${ }^{93} \mathrm{Nb}(90.0 ; 0.41),{ }^{94} \mathrm{Nb}(10.0 ; 4.22)$ \\
$\mathrm{Tc}$ & ${ }^{98} \mathrm{Tc}(8 \mathrm{E}-4$, not available $),{ }^{99} \mathrm{Tc}(99.9992 ; 9.32)$ \\
$\mathrm{Pd}$ & $104 \mathrm{Pd}(18.75 ; 0.66),{ }^{105} \mathrm{Pd}(32.84 ; 3.79), 106 \mathrm{Pd}(15.54 ; 0.28), 107 \mathrm{Pd}(19.47 ; 2.79)$, \\
$\mathrm{I}$ & $108 \mathrm{Pd}(13.38 ; 7.08),{ }^{109} \mathrm{Pd}(0.02 ; 0.28)$ \\
$\mathrm{Cs}$ & ${ }^{127} \mathrm{I}(22.98 ; 4.89),{ }^{129} \mathrm{I}(77.02 ; 3.12)$ \\
$\mathrm{Sm}$ & ${ }^{133} \mathrm{Cs}(76.41 ; 10.6),{ }^{134} \mathrm{Cs}(0.292 ; 11.3),{ }^{135} \mathrm{Cs}(16.83 ; 2.48),{ }^{137} \mathrm{Cs}(6.47 ; 0.03)$ \\
& ${ }^{150} \mathrm{Sm}(63.90 ; 14.8),{ }^{151} \mathrm{Sm}(2.55 ; 660),{ }^{152} \mathrm{Sm}(26.27 ; 74.5),{ }^{154} \mathrm{Sm}(7.28 ; 1.51)$ \\
\hline
\end{tabular}

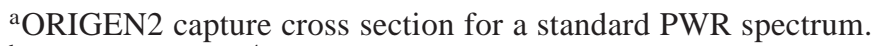

${ }^{\mathrm{b}} \mathrm{Read}$ as $8 \times 10^{-4}$. 
to ${ }^{128} \mathrm{Xe}$ with a half-life of $25 \mathrm{~min}$. Technetium-99 requires no isotopic separation.

Another important factor to be considered in LLFP transmutation is the impact on the geologic repository. This impact of LLFP transmutation on the repository can be measured by the reduction of the leakage-dose risks to biosphere. It is well known that ${ }^{129} \mathrm{I}$ and ${ }^{99} \mathrm{Tc}$ are major contributors to the biosphere release dose rates because of their high geochemical mobility. Technetium-99 and ${ }^{129}$ I are well soluble in the groundwater, and they are hardly sorbed by the geological rocks. The LLFP leakage rate from a repository highly depends on its design of engineering barriers and the geological properties.

Table IV summarizes the transmutation needs of the six transmutable LLFPs in terms of radiotoxicity, halflife, mobility, and inventory and the isotope separation requirement. Note that the dose risk is based on the current evaluation for direct disposal of spent fuel. In terms of reduction of the collective leakage-dose risks (products of toxicity and mobility) in the repository, the transmutation priority can be ranked in the order of ${ }^{129} \mathrm{I},{ }^{99} \mathrm{Tc}$, ${ }^{135} \mathrm{Cs}$, and ${ }^{94} \mathrm{Nb}$. The transmutation need is not high for ${ }^{151} \mathrm{Sm}$ due to its relatively short half-life and for ${ }^{107} \mathrm{Pd}$ due to its low toxicity and low repository dose rate. However, for the effective transmutation of ${ }^{135} \mathrm{Cs}$, isotopic separation is strongly required, and a very high neutron flux $\left(>1.0 \times 10^{16} \mathrm{n} / \mathrm{cm}^{2} \cdot \mathrm{s}\right)$ is necessary even in a thermal spectrum. Consequently, without isotopic separation, the practical candidates for LLFPs transmutation would be ${ }^{129} I$ and ${ }^{99} \mathrm{Tc}$.

The most problematic isotopes, ${ }^{99} \mathrm{Tc}$ and ${ }^{129} \mathrm{I}$, can be transmuted to short-lived or stable isotopes via a single neutron capture reaction. A neutron capture of ${ }^{99} \mathrm{Tc}$ forms ${ }^{100} \mathrm{Tc}$, which shortly decays to stable ${ }^{100} \mathrm{Ru}$ with a half-life of $15.8 \mathrm{~s}$. The daughter nuclide ${ }^{100} \mathrm{Ru}$ is transmuted only to stable or short-lived nuclides by further neutron capture. The capture cross sections of ${ }^{99} \mathrm{Tc}$ and ${ }^{100} \mathrm{Ru}$ are inversely proportional to the neutron velocity in the thermal energy range. Technetium- 99 has a large resonance at $5.6 \mathrm{eV}$ and a series of smaller reso-

\section{TABLE IV}

Transmutation Needs and Isotope Separation Requirement of Transmutable LLFPs

\begin{tabular}{|c|c|c|c|c|c|}
\hline \multirow[b]{2}{*}{ Isotope } & \multicolumn{4}{|c|}{ Transmutation Needs } & \multirow{2}{*}{$\begin{array}{c}\text { Isotope } \\
\text { Separation } \\
\text { Requiremen }\end{array}$} \\
\hline & Toxicity & Half-Life & $\begin{array}{l}\text { Repository } \\
\text { Impact }\end{array}$ & Inventory & \\
\hline${ }^{94} \mathrm{Nb}$ & High & High & Low & Very low & Weak \\
\hline${ }^{99} \mathrm{Tc}$ & Medium & High & High & High & No \\
\hline${ }^{107} \mathrm{Pd}$ & Low & High & Low & Medium & Strong \\
\hline${ }^{129} \mathrm{I}$ & Medium & High & Very high & Medium & Weak \\
\hline${ }^{135} \mathrm{Cs}$ & Medium & High & Medium & Medium & Strong \\
\hline${ }^{151} \mathrm{Sm}$ & High & Low & Low & Low & Weak \\
\hline
\end{tabular}

nances at higher energies: The resonance integral is $\sim 340 \mathrm{~b}$. This indicates that ${ }^{99} \mathrm{Tc}$ might be effectively transmuted in the epithermal energy range. Since ${ }^{100} \mathrm{Ru}$ has a much smaller capture cross section than ${ }^{99} \mathrm{Tc},{ }^{99} \mathrm{Tc}$ would be mainly transmuted to ${ }^{100} \mathrm{Ru}$ with slow conversion rates into ${ }^{101} \mathrm{Ru}$ or ${ }^{102} \mathrm{Ru}$. This also implies that ${ }^{99} \mathrm{Tc}$ transmutation results in a reactivity increase, although it might be quite small.

Since the half-lives of the unstable nuclides ${ }^{128} \mathrm{I}$, ${ }^{130} \mathrm{I},{ }^{131} \mathrm{I}$, and ${ }^{132} \mathrm{I}$ are very short, ${ }^{127} \mathrm{I}$ and ${ }^{129} \mathrm{I}$ are primarily converted by neutron capture to ${ }^{128} \mathrm{Xe}$ and ${ }^{130} \mathrm{Xe}$, respectively. The conversion of ${ }^{127} \mathrm{I}$ to ${ }^{129} \mathrm{I}$ would be very small since ${ }^{128} \mathrm{I}$ quickly decays to ${ }^{128} \mathrm{Xe}$. As a result, both ${ }^{127} \mathrm{I}$ and ${ }^{129} \mathrm{I}$ are transmuted into stable xenon isotopes through neutron capture reactions. In the thermal energy range, the capture cross sections of ${ }^{127} \mathrm{I},{ }^{129} \mathrm{I}$, and ${ }^{130} \mathrm{Xe}$ are inversely proportional to the neutron velocity, but ${ }^{129} \mathrm{I}$ has a much larger capture cross section than the other two isotopes. Iodine-129 has a series of resonances, but they are much smaller than those of ${ }^{99} \mathrm{Tc}$; the resonance integral is $\sim 36 \mathrm{~b}$. Xenon- 130 has a significantly smaller capture cross section than that of ${ }^{129} \mathrm{I}$. Thus, ${ }^{129}$ I target burnup in a thermal spectrum would increase the reactivity.

With respect to the transmutation of ${ }^{129} \mathrm{I}$ and ${ }^{99} \mathrm{Tc}$ in nuclear reactors, several target material forms for ${ }^{99} \mathrm{Tc}$ and ${ }^{129} \mathrm{I}$ transmutation have been studied. Typically, metallic targets have been proposed for ${ }^{99} \mathrm{Tc}$ transmutation, and iodide forms ( $\mathrm{NaI}, \mathrm{CaI}_{2}, \mathrm{CeI}_{3}, \mathrm{PbI}_{2}, \mathrm{CeI}_{3}, \mathrm{YI}_{3}$, etc.) have been considered for ${ }^{129} \mathrm{I}$. Technetium can also be loaded homogeneously by comingling it with the fuel, unlike iodine, which is difficult to mix with the fuel. Irradiation tests performed in the High Flux Reactor at Petten concluded that there is no technical limitation to the use of metallic technetium and that sodium iodide seems to be the best candidate among three iodine target forms considered $\left(\mathrm{NaI}, \mathrm{CeI}_{3}\right.$, and $\left.\mathrm{PbI}_{2}\right)$ from the point of view of comparability with 15-15 titanium stainless steel cladding and with reprocessing technology ${ }^{6-10}$ It has also been reported that sodium iodide $(\mathrm{NaI})$ and calcium iodide $\left(\mathrm{CaI}_{2}\right)$ are desirable target forms for ${ }^{129} \mathrm{I}$ in terms of their chemical characteristics. ${ }^{10} \mathrm{Also}, \mathrm{CeI}_{3}$ and $\mathrm{YI}_{3}$ have favorable properties such as good thermodynamic stability and high iodine density, but fabrication is difficult because of their instability toward oxygen and moisture (i.e., easy formation of oxyiodide). For the $\mathrm{NaI}$ form, a potential concern is that the sodium may melt when it is liberated from the target as the iodine is transmuted to xenon gas.

\section{TRANSMUTATION OF ${ }^{99}$ Tc AND ${ }^{129}$ I IN A PRESSURIZED WATER REACTOR}

Since capture cross sections are high in thermal systems compared to fast systems, it is intuitively 
considered that LLFPs could be efficiently incinerated in a PWR core. However, it seems that there is no general agreement on the LLFP transmutation capability of PWRs. Some previous studies reported that the transmutation of ${ }^{99} \mathrm{Tc}$ and ${ }^{129} \mathrm{I}$ in a PWR core is not promising due to a low transmutation rate and a large required inventory, in spite of relatively large capture cross sections of the LLFPs (Ref. 3). This is mainly due to the low flux level in the PWR core. On the other hand, a recent related study indicated that PWRs have some potential to transmute the LLFPs effectively. ${ }^{6}$ However, these previous studies employed relatively simple models and methods, and focused on limited aspects of the LLFP transmutation. Therefore, the full LLFP transmutation potential of PWRs is reevaluated in a detailed and consistent manner in this study.

Current PWR cores are well optimized in terms of the neutronic and thermal-hydraulic design point of view. Therefore, it is desirable to select LLFP loading options in such a way that the fuel assembly and core design changes due to LLFP loading be minimized. In particular, the maximum loading feasible should be searched for under the design criteria on the feed enrichment, power peaking, fuel burnup, and the available space limit for LLFP loading. For example, the amount of LLFP loading in the conventional $\mathrm{UO}_{2}$ fuel PWR is constrained by the uranium enrichment limit of $5.0 \mathrm{wt} \%{ }^{235} \mathrm{U}$ typically imposed by enrichment plants. More importantly, the negative impacts of LLFPs on the core performance and safety parameters (e.g., cycle length, reactivity swing, reactivity coefficients, rod worths, etc.) should be minimized. Under these considerations, various loading options were investigated for ${ }^{99} \mathrm{Tc}$ and ${ }^{129} \mathrm{I}$, which were identified to have the highest transmutation priorities in Sec. II.

Metal pins were selected as the ${ }^{99} \mathrm{Tc}$ target form based on previous studies. As discussed in Sec. II, NaI and $\mathrm{CaI}_{2}$ are reasonable iodine target forms in terms of their chemical characteristics. However, in the case of NaI, the sodium is liberated from iodide when iodine isotopes are transmuted to xenon, and hence its explosive reaction with water coolant might be a problem at abnormal conditions. Therefore, $\mathrm{CaI}_{2}$ was selected as the ${ }^{129} \mathrm{I}$ target form. To avoid the expense of isotopic separation, the iodide target is directly formed with the elemental iodine extracted from the spent nuclear fuel, which includes both ${ }^{129} \mathrm{I}(77 \%)$ and ${ }^{127} \mathrm{I}(23 \%)$ FPs.

In this section, the maximum LLFP transmutation capability of PWRs under typical design constraints is investigated. The analysis is mainly focused on the standard $\mathrm{UO}_{2}$ fuel assembly, and $\mathrm{UO}_{2}$ and $\mathrm{PuO}_{2}$ mixedoxide (MOX) fuel assembly cases are included for comparison purposes. In Sec. III.A, the calculation models and methods are described. LLFP target pin designs and PWR-based LLFP stabilizer and burner designs are discussed in Secs. III.B and III.C, respectively, and the impacts of LLFP loading on major safety parameters are discussed in Sec. III.D.

\section{III.A. Computational Models and Methods}

Typically, PWR core analyses are done in two steps: Assembly calculations are first performed to generate the few-group homogenized group constants, and then full core neutronics and depletion calculations using these few-group data follow. Based on previous work that demonstrated good agreement between assembly-level and full-core analyses for calculating the heavy metal mass flows in a homogeneous MOX assembly, ${ }^{14}$ assembly calculations with reflective boundary conditions were utilized to estimate the LLFP transmutation performance of PWRs. Since the average behavior of a PWR core can be accurately analyzed by assembly-level analyses when the core is loaded with the same type of fuel assemblies, it is considered that the core-averaged LLFP transmutation rates are estimated with reasonable accuracy by these assembly calculations.

For a standard $17 \times 17$ Westinghouse-type PWR assembly with 24 control rod guide tubes and a central instrument tube, unit assembly calculations were performed using the WIMS8 assembly analysis code ${ }^{15}$ with a 172-group master library. The assembly configuration is shown in Fig. 1, and the assembly design parameters are provided in Table V. The reference PWR conditions for these calculations are also summarized in Table VI. It is assumed that all the fuel pins have the same fuel enrichment, as is the usual case in the Westinghouse fuel assembly design. For MOX fuel assembly analyses, a

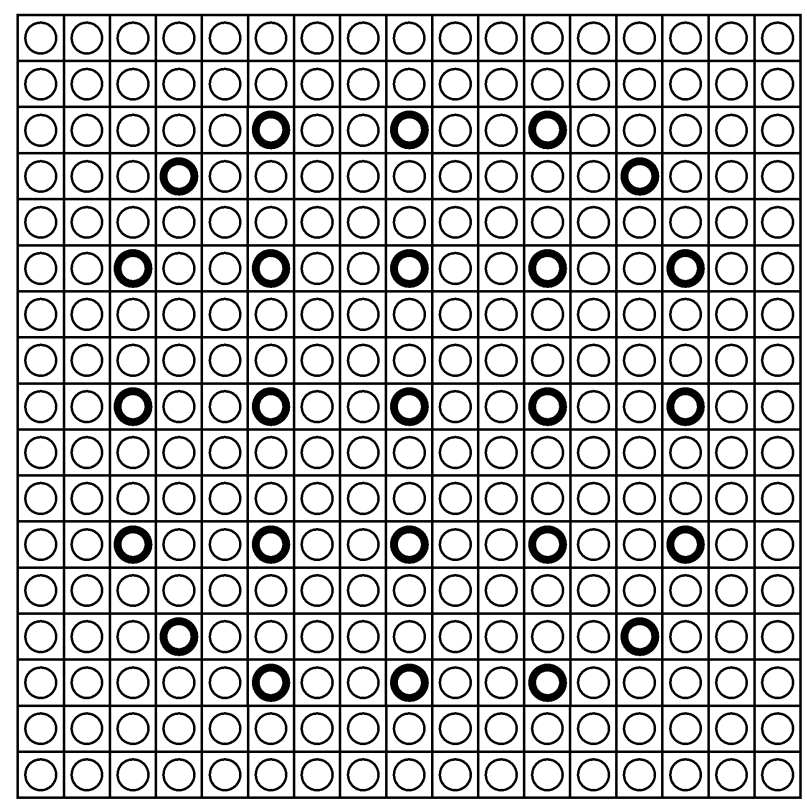

Fuel rod $\quad \mathrm{O}$ Guide tube

Fig. 1. Configuration of $17 \times 17$ Westinghouse-type fuel assembly. 
TABLE V

Design Data of Reference Fuel Assembly

\begin{tabular}{|l|l|}
\hline \multicolumn{1}{|c|}{ Parameter } & Value \\
\hline Pin pitch $(\mathrm{cm})$ & 1.26 \\
Pin diameter $(\mathrm{cm})$ & 0.95 \\
Cladding thickness (cm) & 0.0572 \\
Fuel pellet radius (cm) & 0.4095 \\
Guide-tube outer radius $(\mathrm{cm})$ & 0.6120 \\
Guide-tube thickness $(\mathrm{cm})$ & 0.0405 \\
Interassembly gap $(\mathrm{cm})$ & 0.08 \\
Active assembly height $(\mathrm{cm})$ & 365.76 \\
\hline
\end{tabular}

TABLE VI

Reference Computational Conditions for PWR Fuel Assembly

\begin{tabular}{|l|l|l|c|}
\hline Parameter & \multicolumn{1}{|c|}{ Material } & $\begin{array}{c}\text { Density } \\
\left(\mathrm{g} / \mathrm{cm}^{3}\right)\end{array}$ & $\begin{array}{c}\text { Temperature } \\
(\mathrm{K})\end{array}$ \\
\hline Fuel pellet & $\mathrm{UO}_{2}(\mathrm{MOX})$ & $\overline{-}^{\mathrm{a}}$ & 900 \\
Cladding & Zirconium & 6.50 & 630 \\
Coolant & Water & 0.7053 & 583 \\
Guide tube & Type 303 stainless steel & 7.95 & 600 \\
\hline
\end{tabular}

${ }^{\mathrm{a} C}$ Composition-dependent fuel density.

full-MOX core was assumed with the same fuel management scheme as the $\mathrm{UO}_{2}$ fuel core. A typical light water reactor (LWR) discharge isotopic composition was used for the $\mathrm{PuO}_{2}$ component of the MOX fuel.

In this study, the once-through fuel cycle and a typical three-batch fuel management scheme was assumed with an 18 -month cycle length and an $80 \%$ capacity factor. The power density was set to $37.5 \mathrm{~W} / \mathrm{g} \mathrm{HM}$, which is a typical value of conventional PWRs. The fuel management scheme results in a fuel assembly residence time of 1314 days and an average discharge burnup of $\sim 49 \mathrm{GWd} /$ tonne HM. In assembly depletion calculations, the pinwise depletion option was used, and hence the material density and fluxes are evaluated for individual pins at each depletion time step over the entire irradiation period. Thirty-one depletion time steps were used with variable time intervals; small time steps were used for the early depletion stage to accurately account for the xenon buildup.

In WIMS8 calculations, a solution option based on the method of characteristics was used to solve the twodimensional neutron transport problem. Although the master library is given for a 172-group structure, the two-dimensional transport calculation is performed with 28-group data, which are condensed from a detailed spectral calculation with the 172-group library. It was confirmed that the 28-group results agree well with those of a direct 172-group calculation. The fuel enrichment required for the envisioned fuel management scheme was determined using the linear reactivity model. Assuming that the core leakage effect is $3.5 \% \Delta k$ in a PWR core, the $k_{\text {inf }}$ value should be 1.035 at 876 effective full-power days (EFPD) (equivalent critical burnup state) for the three-batch, 18-month fuel cycle.

In this work, the transmutation rates of LLFPs were evaluated via assembly depletion analysis without accounting for the soluble boron concentration, and the burnable absorbers. In general, the PWR core is maintained critical by adjusting the soluble boron concentration, and a significant amount of excess reactivity is usually controlled by the burnable absorbers. Therefore, for accurate fuel assembly depletion, both the critical soluble boron concentration and the burnable absorbers need to be considered during the depletion calculations. Such thermal neutron absorbers have two opposite impacts on LLFP transmutation. Since they make the neutron spectrum slightly harder, the effective capture cross sections (and hence transmutation rates) of LLFPs are slightly reduced. At the same time, for a fixed power density, they increase the flux level relative to the no-absorber case, thereby enhancing the LLFP transmutation rates. Preliminary analyses showed that the net effects of these absorbers on the LLFP transmutation rate are slightly positive and were neglected in this study for simplicity.

\section{III.B. Long-Lived Fission Product Target Pin Design Study}

Aiming at maximizing the ${ }^{99} \mathrm{Tc}$ and ${ }^{129} \mathrm{I}$ transmutation rates in PWRs under the current design criteria, LLFP target design studies have been performed. In order to minimize the fuel assembly and core design changes, these studies were focused on the LLFP target pin design, with no assembly geometry modification. The option of employing special LLFP target assemblies (distinct from fuel assemblies) was not considered for the PWR study; this option is not favorable since the interior target pins would be exposed to relatively low neutron fluxes because of large shadowing effects of periphery pins.

In order to characterize LLFP transmutation in PWRs, various loading options of ${ }^{99} \mathrm{Tc}$ and ${ }^{129} \mathrm{I}$ that do not require the fuel assembly geometry change were considered using metallic ${ }^{99} \mathrm{Tc}$ and $\mathrm{CaI}_{2}$ (density of $4.52 \mathrm{~g} / \mathrm{cm}^{3}$ ) target forms; for the ${ }^{129} \mathrm{I}$ target, elemental iodine with an isotope vector of $77 \%{ }^{129} \mathrm{I}$ and $23 \%{ }^{127} \mathrm{I}$ was assumed. Since both ${ }^{99} \mathrm{Tc}$ and ${ }^{129} \mathrm{I}$ have relatively large capture cross sections in the thermal and epithermal energy range, a solid cylindrical target form reduces the effective capture cross sections significantly because of spatial selfshielding effects. This is especially true for ${ }^{99} \mathrm{Tc}$ since it has a large capture resonance at $5.6 \mathrm{eV}$. In order to enhance the capture reactions by reducing the spatial selfshielding effects, the LLFP target materials need to be loaded as dilutely as possible. In addition, to take full 
advantage of large thermal capture cross sections of ${ }^{129} \mathrm{I}$ and ${ }^{99} \mathrm{Tc}$, further softening of the neutron spectrum by extra moderation might be desirable. Accordingly, thin annular target forms with homogeneous mixing with fuel or moderator were also considered. In order to investigate the effects of further spectrum softening, $\mathrm{ZrH}_{2}$ (density of $5.61 \mathrm{~g} / \mathrm{cm}^{3}$ ) was chosen as extra moderator since it has a superior moderating power ${ }^{6}$ and a good irradiation experience in TRIGA reactors.

For ${ }^{99} \mathrm{Tc}$, five types of loading options were considered:

1. homogeneous mixing with fuel

2. ${ }^{99} \mathrm{Tc}$ coating of fuel pellet

3. steel-clad annular target loaded in the guide tube

4. annular target material enclosing $\mathrm{ZrH}_{2}$ moderator placed in the guide tube

5. cylindrical target mixed with $\mathrm{ZrH}_{2}$ loaded in the guide tube.

For the ${ }^{129}$ I target, only options 3,4 , and 5 were considered; the options loading ${ }^{129} \mathrm{I}$ inside the fuel cladding were excluded because of the production of gaseous xenon. Figure 2 shows the schematic views of these LLFP loading options.

The self-shielding effects could be minimized by mixing ${ }^{99} \mathrm{Tc}$ with fuel homogeneously since ${ }^{99} \mathrm{Tc}$ could be loaded very dilutely. In addition, the high thermal conductivity of ${ }^{99} \mathrm{Tc}$ may enhance the thermal conductivity of fuel. On the other hand, formation of $\mathrm{TcO}_{2}$ might be a potential problem since it sublimes at $1000 \mathrm{~K}$, even though no serious problem is anticipated when a small amount of ${ }^{99} \mathrm{Tc}$ is mixed with fuel. Thin ${ }^{99} \mathrm{Tc}$ film coating of fuel would reduce the self-shielding effects, but fuel fabrication might be costly. Based on the previous ${ }^{99} \mathrm{Tc}$ irradiation tests, it is expected that there would be no material compatibility problem between ${ }^{99} \mathrm{Tc}$ and cladding. When annular target pins are loaded in guide tubes, the available space for LLFP loading is limited because a significant fraction of the locations must be reserved for control rod insertion. In addition, these options cannot reduce the self-shielding effects as effectively as the first two dilute loading options. It is also not clear whether LLFP target materials are compatible with $\mathrm{ZrH}_{2}$ moderator. On the other hand, LLFP target pins could be fabricated easily, and they can be managed independently of the fuel.

For the above five LLFP loading schemes, WIMS8 calculations were done for both $\mathrm{UO}_{2}\left(4.5 \mathrm{wt} \%{ }^{235} \mathrm{U}\right)$ and full-MOX $\left(10.9 \mathrm{wt} \% \mathrm{PuO}_{2}\right)$ fuel assemblies. Figures 3 and 4 show the effective one-group capture cross sections of ${ }^{99} \mathrm{Tc}$ and ${ }^{129} \mathrm{I}$, respectively, for the various loading options. The neutron capture reaction rates of ${ }^{99} \mathrm{Tc}$ and ${ }^{129} \mathrm{I}$ are presented in Figs. 5 and 6, respectively.

The results in Figs. 3 and 4 show that the capture cross sections of ${ }^{99} \mathrm{Tc}$ and ${ }^{129} \mathrm{I}$ increase monotonically as the LLFP loading decreases; the capture cross section of
${ }^{99} \mathrm{Tc}$ increases almost exponentially with loading decrease, while the ${ }^{129} \mathrm{I}$ cross section increases linearly. The increase of the ${ }^{99} \mathrm{Tc}$ cross section is mainly due to the reduced self-shielding of the large resonance peak at $5.6 \mathrm{eV}$. When the ${ }^{99} \mathrm{Tc}$ loading is significant, the loading options of homogeneous mixing with fuel and ${ }^{99} \mathrm{Tc}$ coating of the fuel pellet give slightly larger cross sections than all others. As the loading decreases, the resonance self-shielding effect decreases, but the spectral softening effect due to extra moderation increases. As a result, for a relatively small ${ }^{99} \mathrm{Tc}$ loading, the cross sections of the cases using additional moderator become larger than the others due to increased spectral softening effect.

In the case of ${ }^{129} \mathrm{I}$, spectral softening is the main cause of cross-section increase, and the resonance selfshielding effect is relatively insignificant. For the same loading of ${ }^{129} \mathrm{I}$, the effective cross sections can be very different depending on the spectrum as shown in Fig. 4. However, the cross-section variation is relatively small compared with that of ${ }^{99} \mathrm{Tc}$ since the neutron spectrum of a typical PWR assembly is already very soft. The use of $\mathrm{ZrH}_{2}$ moderator increases the capture cross section due to the higher moderating power of $\mathrm{ZrH}_{2}$ relative to water; a homogeneous mixture of target and $\mathrm{ZrH}_{2}$ provides a slightly larger value than the annular target enclosing the moderator because of smaller self-shielding effect. Comparing $\mathrm{UO}_{2}$ and MOX fuel assemblies, the harder spectrum in a MOX assembly makes the capture cross sections of both ${ }^{99} \mathrm{Tc}$ and ${ }^{129} \mathrm{I}$ significantly smaller than those of the $\mathrm{UO}_{2}$ assembly, by $\sim 15$ and $\sim 50 \%$, respectively.

In general, these results indicate that the target isotope loading needs to be minimized in order to maximize the effective capture cross section. Because the fractional consumption is directly proportional to the effective onegroup cross section, it is desirable to minimize the target material loading within limits imposed by transmutation goals. However, as shown in Figs. 5 and 6, the capture rates decrease monotonically as the target material loading decreases, even though the effective cross sections increase. As a result, it is necessary to maximize the target material loading under various design constraints if the goal is to maximize the absolute consumption rate.

\section{III.C. Pressurized Water Reactor-Based Long-Lived Fission Product Stabilizer and Burner}

The effective capture cross sections and capture reaction rates discussed in Sec. III.B indicate that in thermal systems, the fractional consumption and absolute consumption rate are somewhat contradictory objectives that cannot be optimized at the same time. Therefore, the targeted mission of LLFP transmutation needs to be defined first, and the performance objective needs to be specified accordingly. In this work, two approaches for LLFP transmutation were postulated. In an LLFP burner 


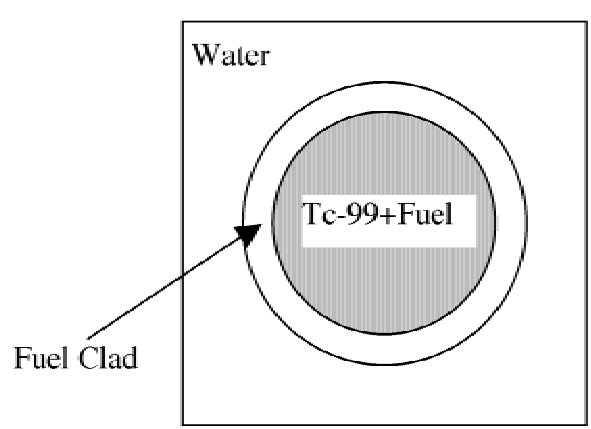

(a) Tc-99 Mixed with Fuel

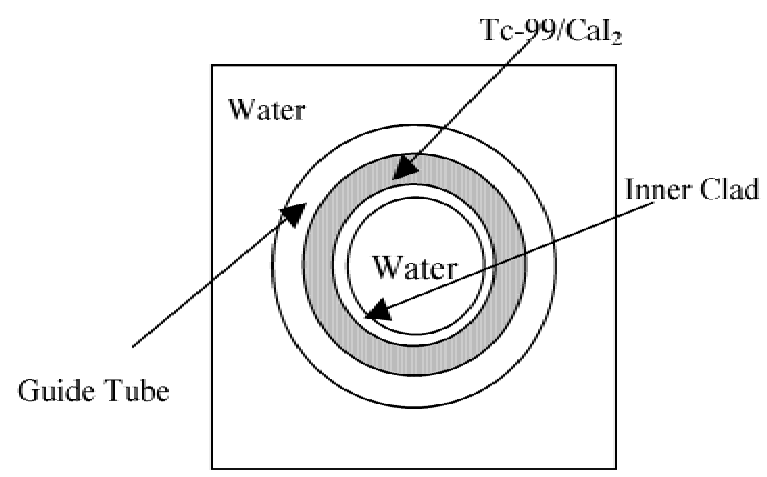

(c) Annular Target in Guide Tube (Inner clad thickness $=0.03 \mathrm{~cm}$ )

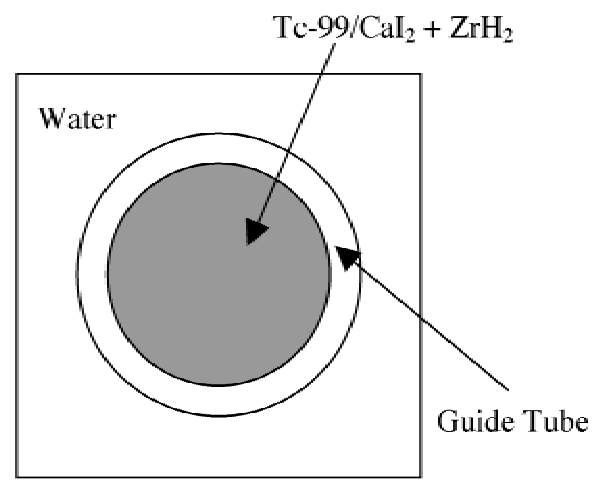

(e) Cylindrical Target Mixed with $\mathrm{ZrH}_{2}$

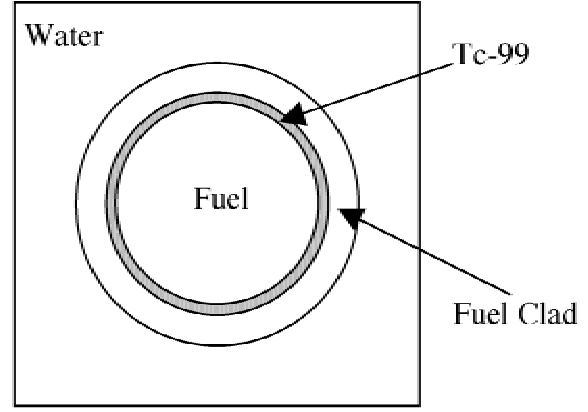

(b) Tc-99-Coated Fuel Pellet

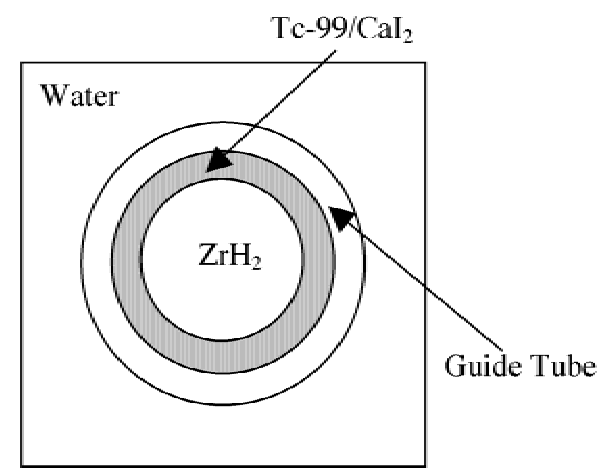

(d) Annular Target with Inner $\mathrm{ZrH}_{2}$

Fig. 2. Target designs for LLFP loading in PWRs.

aimed at burning the accumulated LLFP as quickly (or much) as possible, the maximum consumption rate is of interest. Here one uses the maximum loading of target material that would not degrade core performance and safety parameters. Alternatively, the reactor core could be used only for preventing the buildup of (stabilizing) the LLFP such that only reprocessing losses are sent to the repository. In this LLFP stabilizer, the minimal LLFP loading is determined such that LLFP mass produced is equal to that consumed in a given recycle stage. At as- sembly discharge, the fuel and LLFP target pins are reprocessed, and the recovered LLFPs are fabricated into target pins (or mixed with fuel) and reintroduced.

The feasibility of employing a PWR as an LLFP stabilizer or burner was evaluated by performing depletion calculations for the ${ }^{99} \mathrm{Tc}$ and ${ }^{129} \mathrm{I}$ transmutation rates. The initial loading in the stabilizer case was determined such that the LLFPs produced in the core by fission are balanced by the amount destroyed by neutron capture. In the burner case, since the amount of LLFP destroyed 


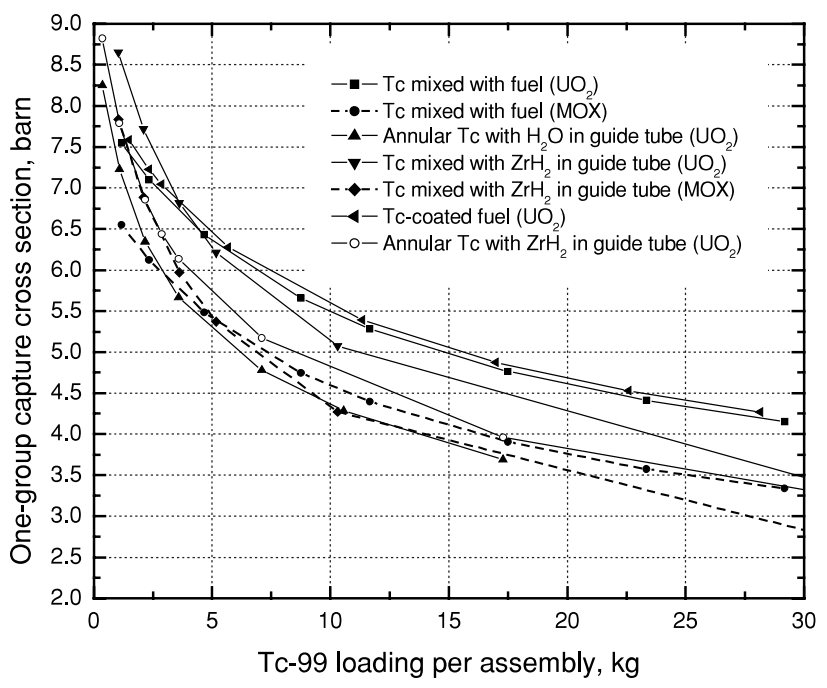

Fig. 3. Effective one-group capture cross section of ${ }^{99} \mathrm{Tc}$ in PWR target assemblies.

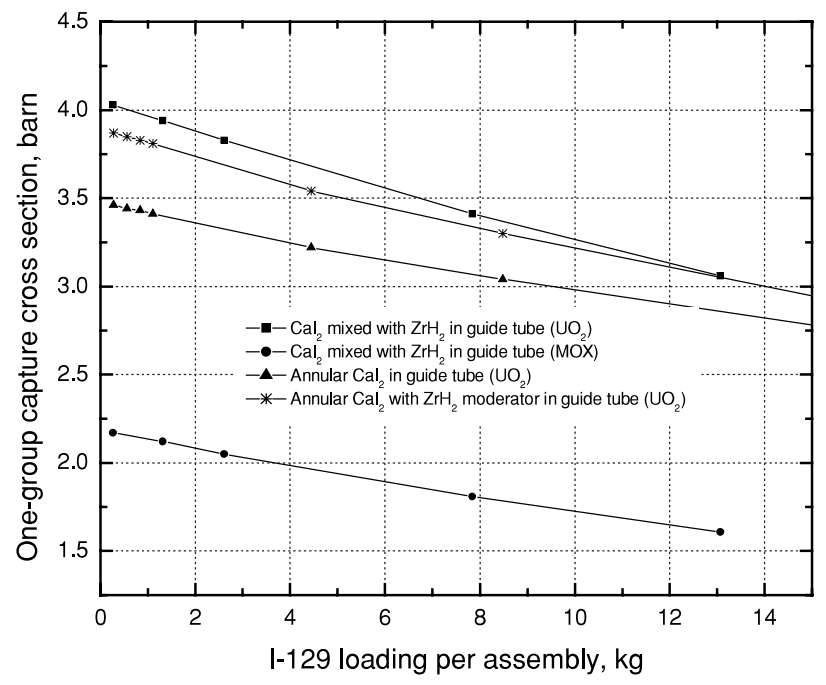

Fig. 4. Effective one-group capture cross section of ${ }^{129} \mathrm{I}$ in PWR target assemblies.

increases with the initial loading, the LLFP loading was maximized under the uranium enrichment limit of 5.0 wt $\%{ }^{235} \mathrm{U}$. A specific power density of $37.5 \mathrm{~W} / \mathrm{g} \mathrm{HM}$ was assumed, and an 18-month cycle length (80\% capacity factor) was used. In each case, the fuel enrichment or the LLFP loading was adjusted such that $k_{\text {inf }}$ is 1.035 at 876 EFPD (equivalent critical burnup state) for a threebatch fuel management scheme. For the cases in which LLFPs are loaded in the guide tube, it was assumed that $40 \%$ of the locations were reserved for control rods. Therefore, a stabilizer assembly should destroy the LLFPs produced in the assemblies where LLFP targets are not loaded, in addition to the self-generated LLFPs. For a

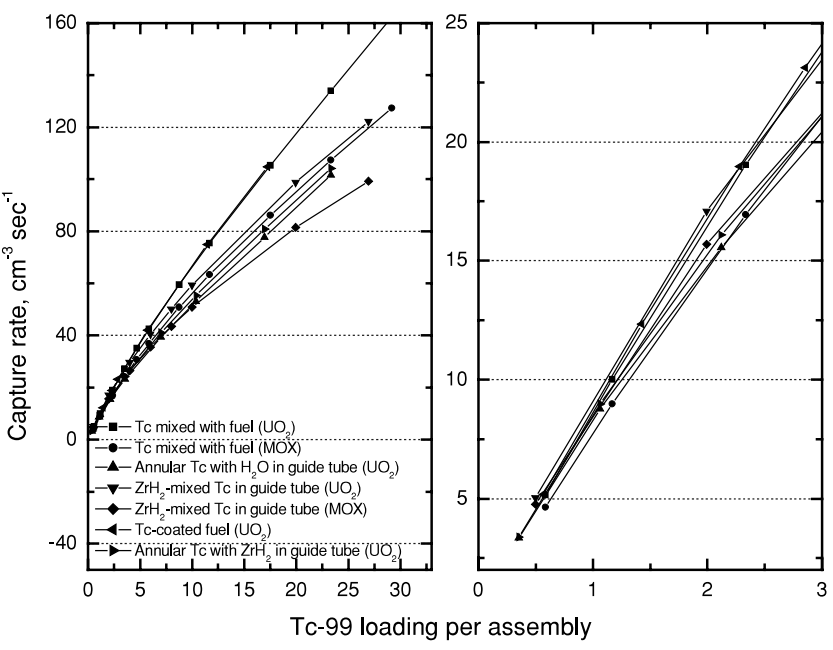

Fig. 5. Capture reaction rate of ${ }^{99} \mathrm{Tc}$ in PWR target assemblies.

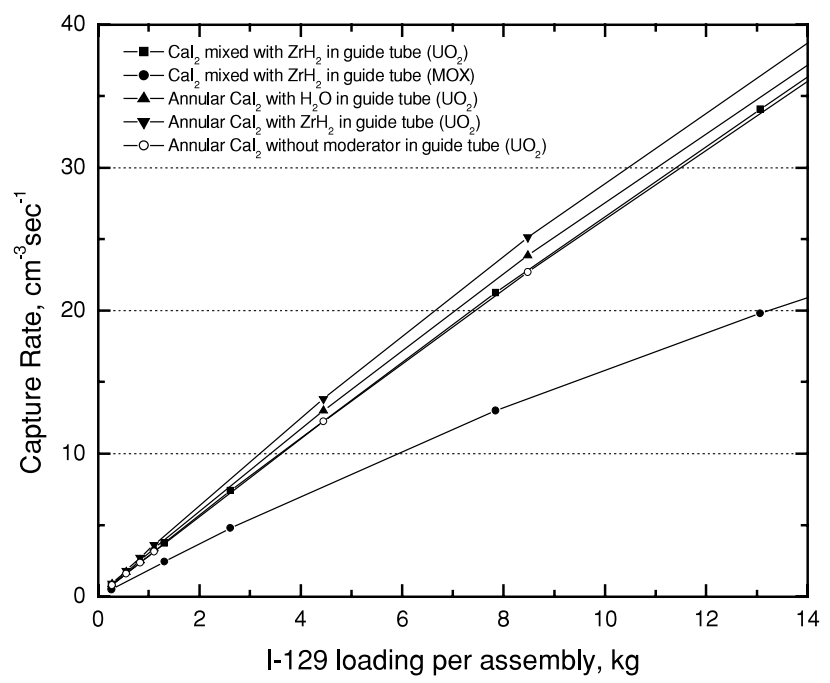

Fig. 6. Capture reaction rate of ${ }^{129} \mathrm{I}$ in PWR target assemblies.

$\mathrm{UO}_{2}$ fuel assembly, these excess quantities amount to $\sim 0.35 \mathrm{~kg}$ /assembly for ${ }^{99} \mathrm{Tc}$ and $\sim 0.083 \mathrm{~kg}$ /assembly for ${ }^{129} \mathrm{I}$, respectively. In the case of a MOX fuel assembly, $\sim 0.35 \mathrm{~kg}$ of ${ }^{99} \mathrm{Tc}$ and $\sim 0.11 \mathrm{~kg}$ of ${ }^{129}$ I need to be additionally destroyed per assembly.

The resulting transmutation performances are summarized in Table VII. For the cases of homogeneous mixing of ${ }^{99} \mathrm{Tc}$ and fuel, the production rate was approximated by the production rate of the corresponding Tccoated fuel pellet case for the $\mathrm{UO}_{2}$ fuel and by that of the base case for the MOX fuel, since the WIMS8 code does not distinguish the ${ }^{99} \mathrm{Tc}$ nuclides produced by fission from the initial target nuclides. Table VII shows that 
TABLE VII

Transmutation Performance of ${ }^{99} \mathrm{Tc}$ and ${ }^{129} \mathrm{I}$ in PWR Assemblies*

\begin{tabular}{|c|c|c|c|c|c|c|c|}
\hline Case & $\begin{array}{c}{ }^{235} \mathrm{U} \text { or } \mathrm{Pu} \\
\text { Fraction of } \\
\text { Heavy Metal } \\
\quad(\mathrm{wt} \%)\end{array}$ & LLFP & $\begin{array}{l}\text { Initial } \\
\text { Loading } \\
\quad(\mathrm{kg})\end{array}$ & $\begin{array}{l}\text { Production } \\
\quad(\mathrm{kg})\end{array}$ & $\begin{array}{l}\text { Net } \\
\text { Consumption } \\
(\mathrm{kg})\end{array}$ & $\begin{array}{l}\text { LLFP } \\
\text { Discharge } \\
\text { Burnup } \\
\text { (at.\%) }\end{array}$ & Remarks \\
\hline \multicolumn{8}{|c|}{$\mathrm{UO}_{2}$ Fuel Assembly, LLFP Stabilizer } \\
\hline Base case & 4.38 & ${ }^{99} \mathrm{Tc}$ & $\begin{array}{l}0.0 \\
0.0\end{array}$ & $\begin{array}{l}0.532 \\
0.123\end{array}$ & $\begin{array}{l}-0.532 \\
-0.123\end{array}$ & - & No initial LLFP \\
\hline Tc mixed with fuel & 4.79 & ${ }^{99} \mathrm{Tc}$ & 2.568 & $0.540^{\mathrm{a}}$ & +0.000 & 21.0 & $0.49 \mathrm{wt} \% \mathrm{Tc}$ \\
\hline Tc target mixed with $\mathrm{ZrH}_{2}$ & 5.17 & ${ }^{99} \mathrm{Tc}$ & 4.588 & 0.540 & +0.356 & 19.5 & 95.4 vol\% $\mathrm{ZrH}_{2}$ \\
\hline Tc-coated fuel pellet & 4.79 & ${ }^{99} \mathrm{Tc}$ & 2.511 & 0.540 & -0.004 & 21.7 & $8.8-\mu \mathrm{m} \mathrm{Tc}$ \\
\hline $\mathrm{CaI}_{2}$ target mixed with $\mathrm{ZrH}_{2}$ & 4.58 & ${ }^{129} \mathrm{I}$ & 1.714 & 0.121 & +0.088 & 12.2 & 93.4 vol\% $\mathrm{ZrH}_{2}$ \\
\hline $\begin{array}{l}\text { Tc mixed with fuel and } \mathrm{CaI}_{2} \\
\text { target mixed with } \mathrm{ZrH}_{2}\end{array}$ & 4.99 & $\begin{array}{c}{ }^{99} \mathrm{Tc} \\
{ }^{129} \mathrm{I}\end{array}$ & $\begin{array}{l}2.655 \\
1.777\end{array}$ & $\begin{array}{l}0.540^{\mathrm{a}} \\
0.120\end{array}$ & $\begin{array}{l}-0.001 \\
+0.086\end{array}$ & $\begin{array}{l}20.3 \\
11.6\end{array}$ & $\begin{array}{l}0.50 \mathrm{wt} \% \mathrm{Tc} \\
93.2 \text { vol\% } \mathrm{ZrH}_{2}\end{array}$ \\
\hline \multicolumn{8}{|c|}{$\mathrm{UO}_{2}$ Fuel Assembly, LLFP Burner } \\
\hline $\begin{array}{l}\text { Tc mixed with fuel } \\
\text { Tc-coated fuel pellet } \\
\mathrm{CaI}_{2} \text { target mixed with } \mathrm{ZrH}_{2}\end{array}$ & $\begin{array}{l}5.00 \\
5.00 \\
5.00\end{array}$ & $\begin{array}{l}{ }^{99} \mathrm{Tc} \\
{ }^{99} \mathrm{Tc} \\
{ }^{129} \mathrm{I}\end{array}$ & $\begin{array}{l}3.968 \\
3.882 \\
5.515\end{array}$ & $\begin{array}{l}0.540^{\mathrm{a}} \\
0.544 \\
0.120\end{array}$ & $\begin{array}{l}+0.246 \\
+0.249 \\
+0.471\end{array}$ & $\begin{array}{l}19.8 \\
20.4 \\
10.7\end{array}$ & $\begin{array}{l}0.75 \mathrm{wt} \% \mathrm{Tc} \\
13.6-\mu \mathrm{m} \mathrm{Tc} \\
78.9 \mathrm{vol}^{2} \mathrm{ZrH}_{2}\end{array}$ \\
\hline \multicolumn{8}{|c|}{ MOX Fuel Assembly, LLFP Stabilizer } \\
\hline Base case & 10.8 & ${ }^{99} \mathrm{Tc}$ & $\begin{array}{l}0.0 \\
0.0\end{array}$ & $\begin{array}{l}0.527 \\
0.164\end{array}$ & $\begin{array}{l}-0.527 \\
-0.164\end{array}$ & - & No initial LLFP \\
\hline Tc mixed with fuel & 12.8 & ${ }^{99} \mathrm{Tc}$ & 3.180 & $0.527^{\mathrm{b}}$ & +0.013 & 17.0 & $0.60 \mathrm{wt} \% \mathrm{Tc}$ \\
\hline $\begin{array}{l}\text { Tc mixed with fuel and } \mathrm{CaI}_{2} \\
\text { target mixed with } \mathrm{ZrH}_{2}\end{array}$ & 14.5 & ${ }^{99} \mathrm{Tc}$ & $\begin{array}{l}3.256 \\
4.835\end{array}$ & $\begin{array}{l}0.527^{b} \\
0.165\end{array}$ & $\begin{array}{l}-0.004 \\
+0.125\end{array}$ & $\begin{array}{r}16.1 \\
6.0\end{array}$ & $\begin{array}{l}0.60 \mathrm{wt} \% \mathrm{Tc} \\
81.5 \mathrm{vol} \% \mathrm{ZrH}_{2}\end{array}$ \\
\hline
\end{tabular}

* Masses are per unit assembly, and the active assembly height is $365.76 \mathrm{~cm}$.

${ }^{\text {a Self-generated }}{ }^{99} \mathrm{Tc}$ from the Tc-coated fuel case.

${ }^{\mathrm{b}}$ Self-generated ${ }^{99} \mathrm{Tc}$ from the base case.

in a $\mathrm{UO}_{2}$ core, $\sim 20$ and $\sim 12 \%$ of the initial loading ${ }^{99} \mathrm{Tc}$ and ${ }^{129} \mathrm{I}$, respectively, are consumed at discharge. Additionally, the results indicate that a PWR LLFP stabilizer for ${ }^{99} \mathrm{Tc}$ and/or ${ }^{129} \mathrm{I}$ would be feasible with an increase in uranium enrichment, relative to the base case with no LLFP loading. The uranium enrichment is generally less than the $5 \%$ limit, except for the case using ${ }^{99} \mathrm{Tc}$ target pins mixed with $\mathrm{ZrH}_{2}$. In this case, the required uranium enrichment is slightly higher than $5.0 \mathrm{wt} \%$ because of the larger amount of LLFP loading required to compensate for the assemblies not loaded with target pins. However, this enrichment is artificial because of the assembly model used in this study, and it would be slightly reduced in a full-core calculation. This is similarly valid for the ${ }^{129} \mathrm{I}$ results since ${ }^{129} \mathrm{I}$ targets are loaded in guide tubes only.

The potential of using a PWR as an LLFP burner is limited because the initial LLFP loading is constrained by the uranium enrichment. Nonetheless, results indicate that ${ }^{99} \mathrm{Tc}$ and ${ }^{129} \mathrm{I}$ can be burned with support ratios [de- fined as the number of conventional light water reactors (LWRs) that a burner supports, i.e., the ratio of megawatt(thermal) from LWRs to megawatt(thermal)from a burner] of $\sim 0.5$ and $\sim 3$, respectively, but the low discharge burnup of ${ }^{129} \mathrm{I}$ would require a large number of reprocessing stages for complete burning. The amount of LLFPs incinerated could be increased by maximizing the LLFP loading within the imposed enrichment limit, but this would reduce the cycle length and the LLFP discharge burnup.

In the MOX core, the discharge burnups of ${ }^{99} \mathrm{Tc}$ and ${ }^{129}$ I are smaller by $\sim 19$ and $\sim 50 \%$, respectively, than those for the $\mathrm{UO}_{2}$ core, due to the hardened neutron spectrum (see Figs. 7 and 8). A more efficient LLFP transmutation could be achieved in the MOX-fueled core by increasing the neutron moderation. For example, the highmoderator-ratio ${ }^{16}$ (HMR) reactor concept for a fullMOX core would be helpful in enhancing the LLFP transmutation performance. Nevertheless, it is expected 


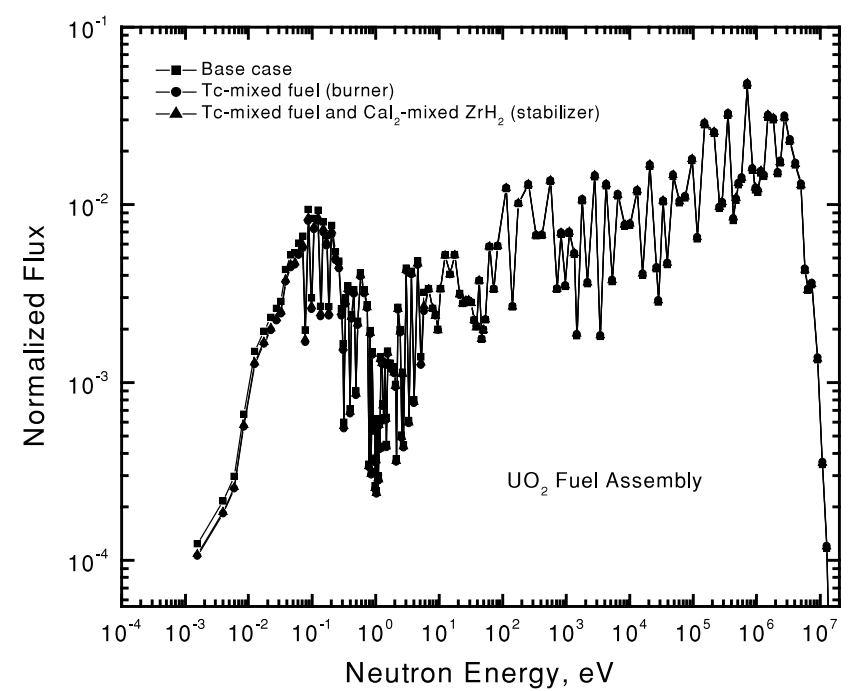

Fig. 7. Neutron spectrum in $\mathrm{UO}_{2}$ fuel target assemblies.

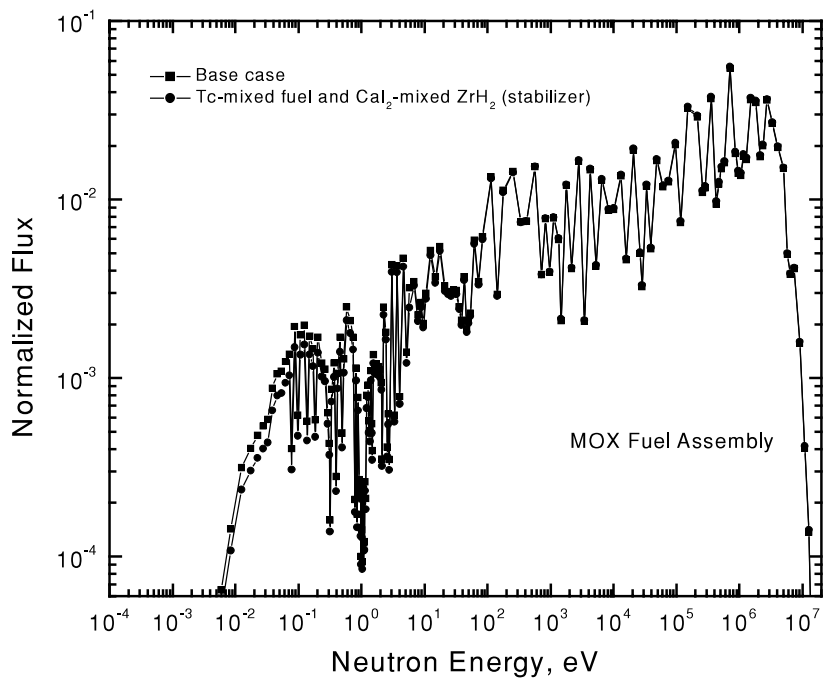

Fig. 8. Neutron spectrum in MOX fuel target assemblies.

that the ${ }^{99} \mathrm{Tc}$ and ${ }^{129} \mathrm{I}$ transmutation performance in the HMR MOX core would not be significantly higher. In the same context, a TRU-loaded PWR core is expected to have LLFP transmutation performance similar to that of the typical PWR loaded with MOX.

Figures 7 and 8, respectively, compare the average neutron spectra (172-group) of $\mathrm{UO}_{2}$ and $\mathrm{MOX}$ assemblies with and without LLFP loading. For both $\mathrm{UO}_{2}$ and MOX fuel assemblies, the neutron spectra of LLFPloaded assemblies are only slightly harder than that of the base case because of thermal neutron captures in LLFP targets. The additional moderator in the guide tube does not affect the assembly average neutron spectrum noticeably since it is confined only to the guide-tube locations. Therefore, it is expected that the neutronic properties of the LLFP-loaded fuel assemblies are not very different from those of the base case without LLFPs. In Figs. 9 and 10, the $k_{\text {inf }}$ value is plotted over the irradiation period for various target configurations. It is observed that ${ }^{99} \mathrm{Tc}$ and ${ }^{129} \mathrm{I}$ transmutation in a PWR reduces the reactivity swing noticeably. This is because ${ }^{99} \mathrm{Tc}$ and ${ }^{129} \mathrm{I}$ are respectively transmuted into $\mathrm{Ru}$ and $\mathrm{Xe}$ elements, which have significantly smaller capture cross sections than their parent isotopes. The reduced reactivity swing indicates that ${ }^{99} \mathrm{Tc}$ and ${ }^{129} \mathrm{I}$ could be used as burnable absorbers although they have relatively large residual reactivity penalties due to limited discharge burnups. The reduced initial excess reactivity in an LLFPloaded PWR may result in a reduced maximum soluble boron concentration.

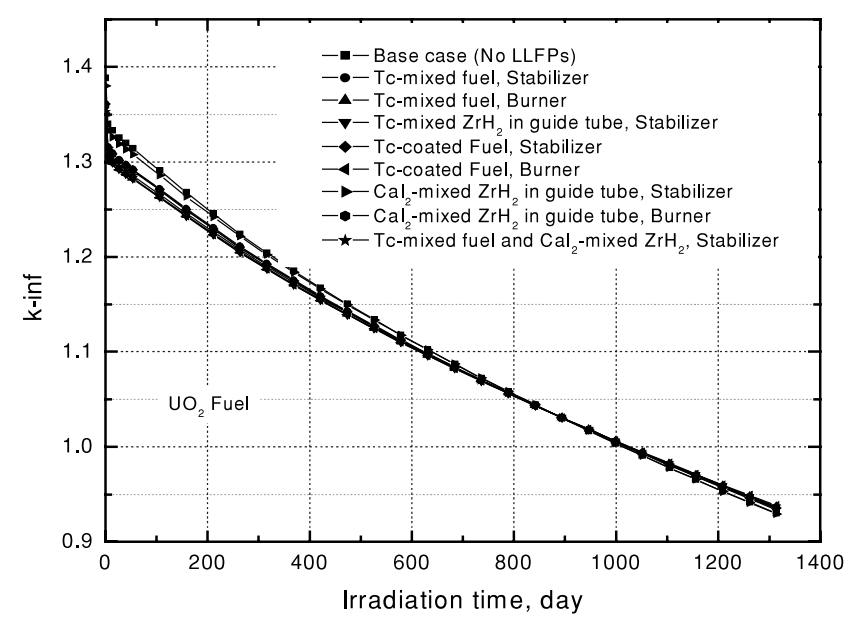

Fig. 9. Infinite multiplication factor of $\mathrm{UO}_{2}$ fuel target assemblies versus irradiation time.

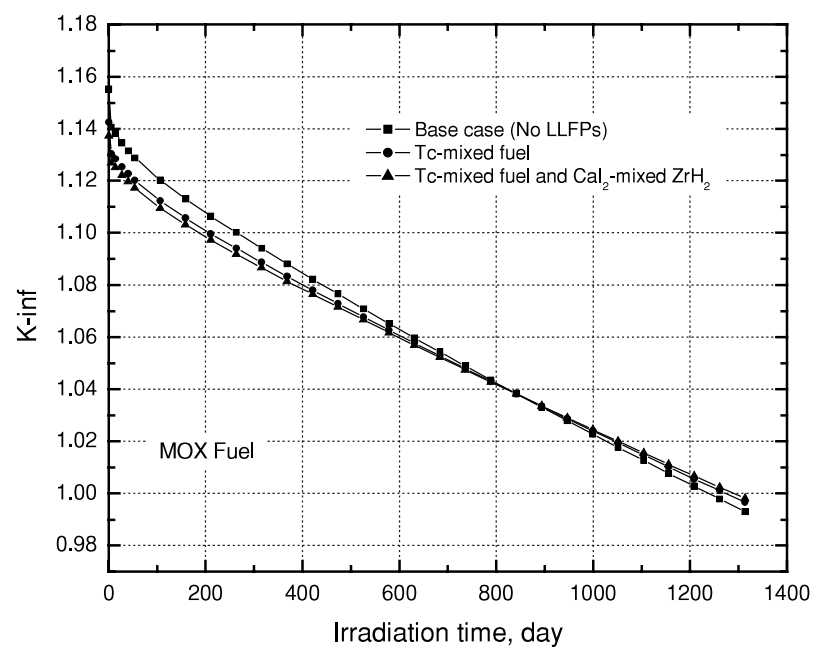

Fig. 10. Infinite multiplication factor of MOX fuel target assemblies versus irradiation time. 
Both ${ }^{127} \mathrm{I}$ and ${ }^{129} \mathrm{I}$ are transmuted mainly into stable isotopes ${ }^{128} \mathrm{Xe}$ and ${ }^{130} \mathrm{Xe}$, respectively. Since these xenon isotopes are in gaseous state, the xenon gas pressure buildup in an iodine target would be one of the limiting factors for the iodine transmutation, if an iodine target is placed in a sealed pin. Therefore, a preliminary evaluation of the xenon gas pressure buildup was performed for a $\mathrm{CaI}_{2}$ target pin using the ideal gas law. The xenon pressure buildup depends on the gas release rate from the iodine target, but no data is available at the moment. Therefore, for a conservative pressure evaluation, it was assumed that all the xenon gas produced in the target is released into the space between the target and cladding. The results are presented in Fig. 11 for various burnup levels of the iodine isotopes as a function of the volume ratio of gas plenum to $\mathrm{CaI}_{2}$ target.

Figure 11 shows that a relatively large gas plenum is required for a high iodine burnup. For example, consider a case of homogeneous mixture of $\mathrm{CaI}_{2}$ and $\mathrm{ZrH}_{2}$ moderator loaded in the guide-tube location, for which the iodine discharge burnup was shown to be $\sim 10$ to 12 at. $\%$ depending on the volume fraction of the moderator. Assuming a $15.25-\mathrm{cm}$ gas plenum as in the typical fuel rod in PWRs, the volume fraction occupied by the target mixture is $\sim 0.93$. If the moderator fraction is 0.8 in the target mixture, the volume ratio of gas plenum to $\mathrm{CaI}_{2}$ target becomes $\sim 0.38$. In this case, the internal xenon pressure is $\sim 540 \mathrm{~atm}$ for a 12 at.\% burnup of iodine. If we take the allowable internal gas pressure of a typical PWR fuel pin, which is $\sim 157$ atm similar to the system pressure, as the maximum allowable xenon pressure, the $\mathrm{CaI}_{2}$ volume should be smaller than $77 \%$ of the gas plenum volume for a 12 at.\% iodine discharge burnup. For the iodine target with an inner moderator, it would be difficult to obtain the required gas plenum space for a

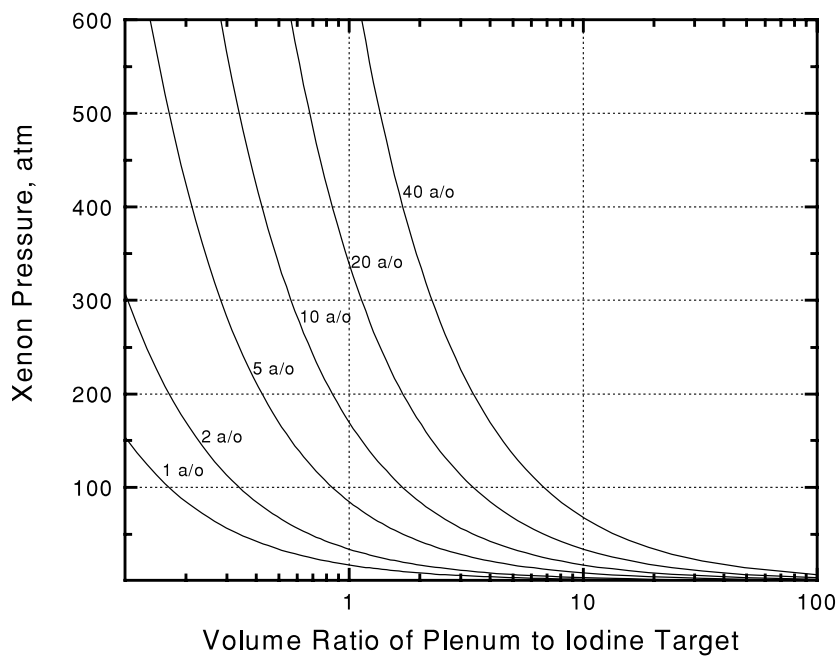

Fig. 11. Xenon gas pressure in $\mathrm{CaI}_{2}$ target pin for various iodine burnup levels $(680 \mathrm{~K})$.
12 at.\% discharge burnup. On the other hand, if an annular iodine target with inner void region is loaded in the guide tube, a sufficient gas plenum space would be available for the 12 at.\% iodine discharge burnup.

\section{III.D. Impacts of Long-Lived Fission Product Loading on Safety Parameters}

In order for LLFPs to be routinely transmuted in PWRs, their loading should not significantly degrade the safety potential of the core. Major safety-related parameters were evaluated for several LLFP loading options and compared with those of the standard $\mathrm{UO}_{2}$ fuel assembly. Specifically, the fuel temperature coefficient (FTC), moderator temperature coefficient (MTC), soluble boron worth, and control rod worth were estimated. Generally, the reactivity coefficients of a PWR core are evaluated for the critical boron concentration. With assembly calculation methodology, however, the critical conditions are not typically searched. Thus, a constant soluble boron concentration of $500 \mathrm{ppm}$ was assumed for this analysis. The reactivity coefficients were calculated for the base case without LLFP loading and for two LLFP-loaded assemblies: the ${ }^{99} \mathrm{Tc}$ burner case and the ${ }^{99} \mathrm{Tc} /{ }^{129} \mathrm{I}$ stabilizer discussed in Sec. III.C. The ${ }^{99} \mathrm{Tc}$ burner was loaded with Tc by homogeneous mixing of Tc with fuel, and Tc loading was maximized within the fuel enrichment limit of $5 \mathrm{wt} \%{ }^{235} \mathrm{U}$. The ${ }^{99} \mathrm{Tc} /{ }^{129} \mathrm{I}$ stabilizer was simultaneously loaded with Tc and I by additionally placing the $\mathrm{CaI}_{2}$ targets mixed $\mathrm{ZrH}_{2}$ in the guide-tube locations.

Figure 12 compares the burnup-dependent FTCs of the LLFP-loaded assemblies with that of the standard assembly. It is observed that FTCs of the LLFP-loaded assemblies are slightly less negative. This is mainly due

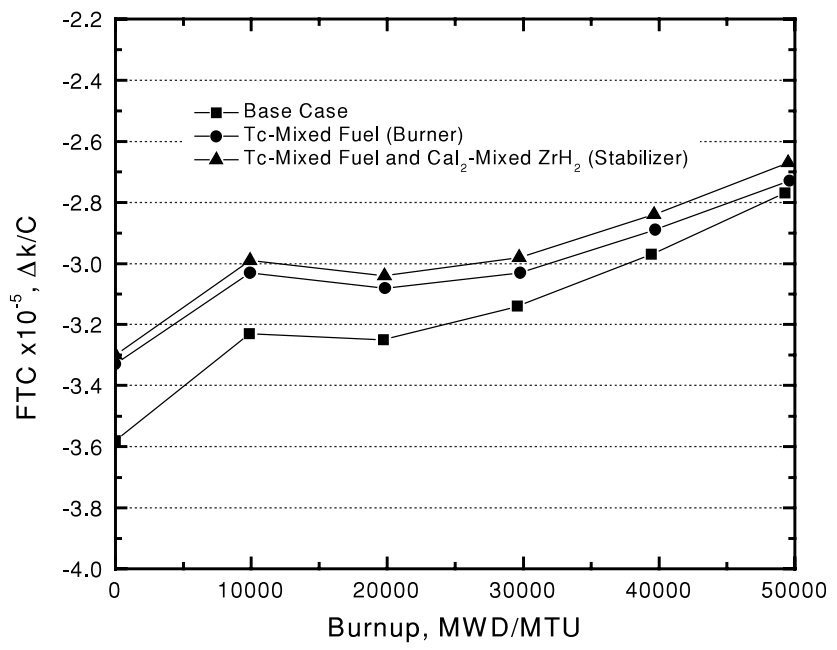

Fig. 12. Burnup-dependent fuel temperature coefficient at $900 \mathrm{~K}$ 
to the higher fuel enrichments of the LLFP-loaded assemblies than the standard one. Figure 13 shows that the MTC of ${ }^{99} \mathrm{Tc} /{ }^{129} \mathrm{I}$ stabilizer is comparable to the base case MTC, while the ${ }^{99} \mathrm{Tc}$ burner has more negative MTC values. For the Tc-burner case, the MTC becomes more negative by $\sim 14 \%$, and it is considered that this amount of change would not cause any safety problem. This enhanced MTC may help the core safety when the soluble boron concentration is high at the beginning of cycle.

Figure 14 shows that the soluble boron worths are slightly reduced in the LLFP-loaded cases compared with the standard assembly. This boron worth reduction is again due to the neutron spectrum hardening caused by LLFP loading. The ${ }^{99} \mathrm{Tc} /{ }^{129} \mathrm{I}$ stabilizer has the smallest

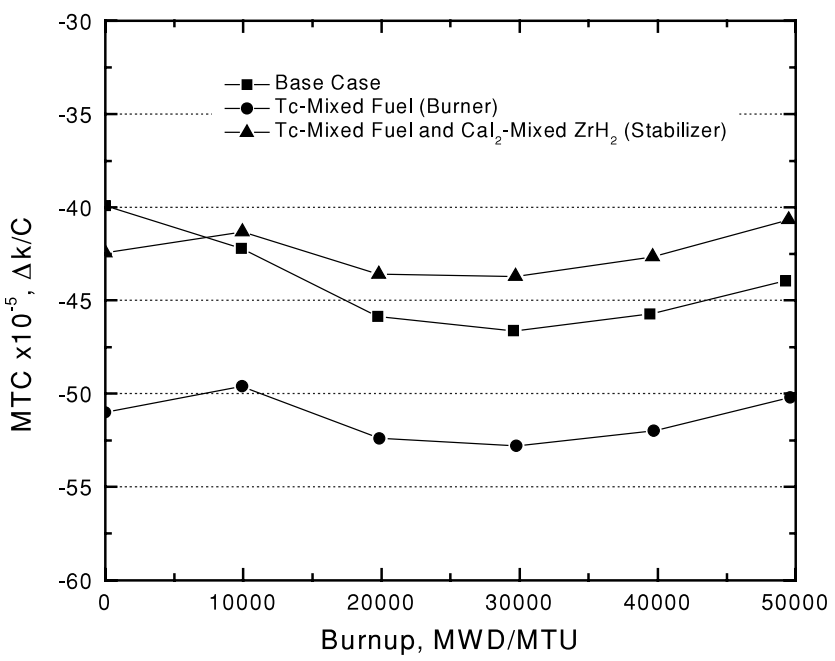

Fig. 13. Burnup-dependent moderator temperature coefficient at $583 \mathrm{~K}$.

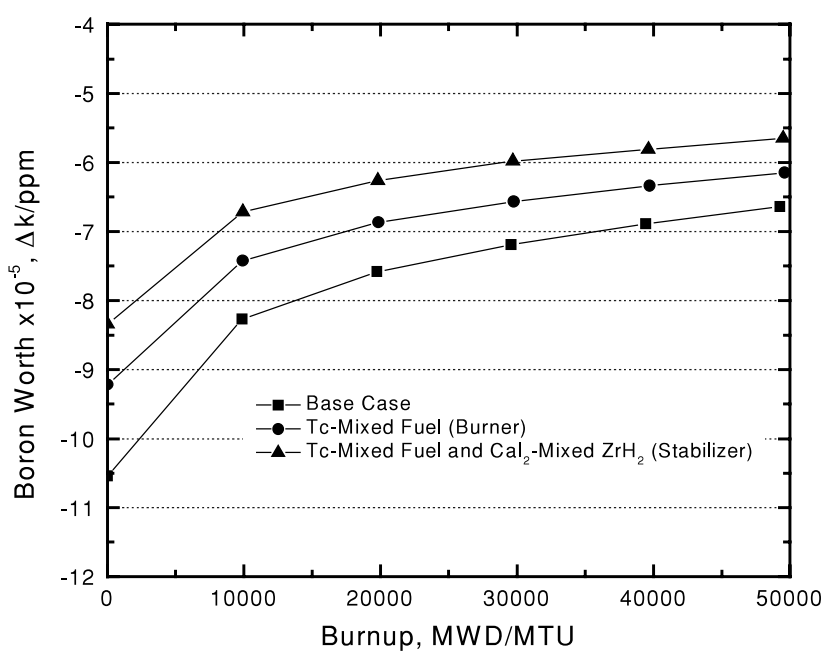

Fig. 14. Burnup-dependent boron worth at $500 \mathrm{ppm}$. boron worth because of the reduced coolant volume. Although the boron worth is slightly smaller in an LLFPloaded assembly, the critical boron concentration in the core should not noticeably increase because the burnup reactivity swing is sufficiently smaller in the LLFPloaded core, as shown in Fig. 9. Generally, the soluble boron worth is a function of the boron concentration, being larger at low concentration. However, it is expected that the trends shown in Fig. 14 would not change noticeably at different boron concentrations.

The control rod worth is compared in Fig. 15 for the standard and Tc-mixed fuel cases only, since the control rod cannot be inserted when LLFPs are loaded in the guide-tube locations. It can be seen that the control rod worth of the LLFP-loaded assembly is marginally decreased (by $\sim 1 \%$ ) again due to the hardened neutron spectrum. Because this change is small, the worth of the control rods might not be an issue in the LLFP-loaded PWR core. Taking into account all these results, it is concluded that ${ }^{99} \mathrm{Tc}$ and $/$ or ${ }^{129}$ I can be transmuted in a $\mathrm{UO}_{2}$-fueled $\mathrm{PWR}$ core without any problem from the viewpoint of reactivity coefficients as long as the LLFP loading is bounded by the current uranium enrichment limit of $5 \mathrm{wt} \%{ }^{235} \mathrm{U}$.

Another important consideration for reactor safety is the power peaking factor. Since the power distribution is not changed when LLFP is uniformly mixed with fuel, the LLFP-induced perturbation in the power distribution was conservatively assessed by considering an assembly loaded with the mixture of $20 \% \mathrm{Tc}$ and $80 \% \mathrm{ZrH}_{2}$ by volume in the guide-tube locations. In this case, $\sim 20 \mathrm{~kg}$ of ${ }^{99} \mathrm{Tc}$ is loaded in an assembly, which is much larger than the ${ }^{99} \mathrm{Tc}$ mass in the ${ }^{99} \mathrm{Tc}$ burner case. Figure 16 compares the normalized pinwise power distribution at zero burnup with that of the standard assembly. It is observed that the power distribution is actually flatter in

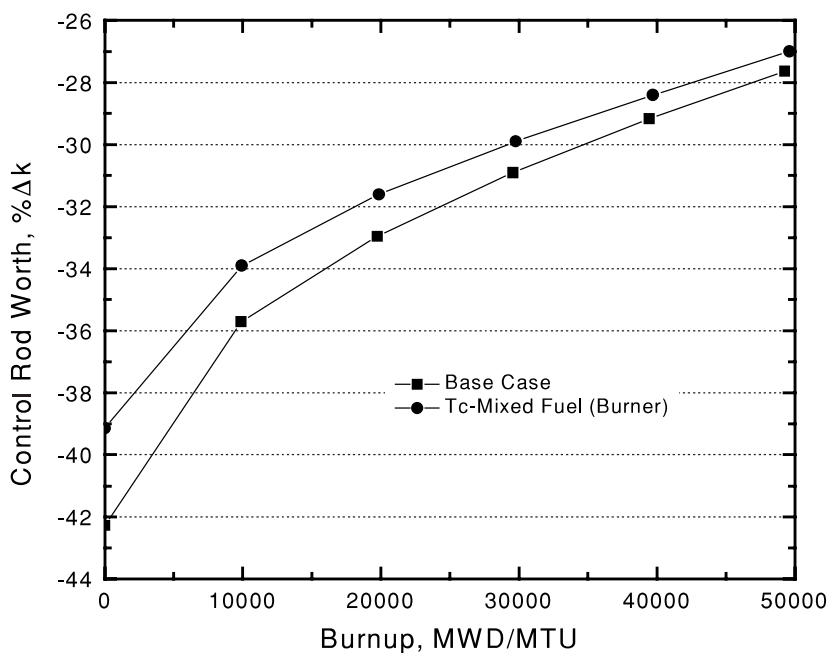

Fig. 15. Burnup-dependent $\mathrm{B}_{4} \mathrm{C}$ control rod worth. 


\begin{tabular}{|c|c|c|c|c|c|c|c|c|}
\hline $\begin{array}{l}0 . \\
0 .\end{array}$ & & & & & & & & \\
\hline $\begin{array}{l}1.039 \\
1.047\end{array}$ & $\begin{array}{l}1.010 \\
1.014\end{array}$ & & & & & & & \\
\hline $\begin{array}{l}1.038 \\
1.009\end{array}$ & $\begin{array}{l}1.012 \\
0.996\end{array}$ & $\begin{array}{l}1.013- \\
0.991-\end{array}$ & & standar & & & & \\
\hline $\begin{array}{l}0 . \\
0 .\end{array}$ & $\begin{array}{l}1.040 \\
0.997\end{array}$ & $\begin{array}{l}1.041 \\
0.994\end{array}$ & $\begin{array}{l}0 . \\
0 .\end{array}$ & & & & & \\
\hline $\begin{array}{l}1.037 \\
0.993\end{array}$ & $\begin{array}{l}1.011 \\
0.985\end{array}$ & $\begin{array}{l}1.014 \\
0.985\end{array}$ & $\begin{array}{l}1.048 \\
0.995\end{array}$ & $\begin{array}{l}1.038 \\
0.994\end{array}$ & & & & \\
\hline $\begin{array}{l}1.034 \\
0.994\end{array}$ & $\begin{array}{l}1.007 \\
0.985\end{array}$ & $\begin{array}{l}1.011 \\
0.987\end{array}$ & $\begin{array}{l}1.048 \\
1.000\end{array}$ & $\begin{array}{l}1.057 \\
1.004\end{array}$ & $\begin{array}{l}0 . \\
0 .\end{array}$ & & & \\
\hline $\begin{array}{l}0 . \\
0 .\end{array}$ & $\begin{array}{l}1.028 \\
0.997\end{array}$ & $\begin{array}{l}1.029 \\
0.998\end{array}$ & $\begin{array}{l}0 . \\
0 .\end{array}$ & $\begin{array}{l}1.041 \\
1.007\end{array}$ & $\begin{array}{l}1.019 \\
1.006\end{array}$ & $\begin{array}{l}0.973 \\
1.000\end{array}$ & & \\
\hline $\begin{array}{l}1.013 \\
0.999\end{array}$ & $\begin{array}{l}0.988 \\
0.993\end{array}$ & $\begin{array}{l}0.989 \\
0.994\end{array}$ & $\begin{array}{l}1.013 \\
1.003\end{array}$ & $\begin{array}{l}0.983 \\
0.999\end{array}$ & $\begin{array}{l}0.960 \\
0.997\end{array}$ & $\begin{array}{l}0.943 \\
0.999\end{array}$ & $\begin{array}{l}0.930 \\
1.003\end{array}$ & \\
\hline $\begin{array}{l}0.973 \\
1.000\end{array}$ & $\begin{array}{l}0.969 \\
0.998\end{array}$ & $\begin{array}{l}0.969 \\
0.999\end{array}$ & $\begin{array}{l}0.971 \\
1.004\end{array}$ & $\begin{array}{l}0.962 \\
1.004\end{array}$ & $\begin{array}{l}0.951 \\
1.007\end{array}$ & $\begin{array}{l}0.941 \\
1.012\end{array}$ & $\begin{array}{l}0.936 \\
1.018\end{array}$ & $\begin{array}{l}0.944 \\
1.033\end{array}$ \\
\hline
\end{tabular}

Fig. 16. Power distributions of standard and Tc-loaded assemblies.

the Tc-loaded assembly than in the standard one. As shown in Fig. 16, the peak power in the standard assembly is 1.057, while it is 1.047 for the Tc-loaded case. This is because the power peaking usually occurs in the vicinity of the guide-tube cells in the standard fuel assembly. In the PWR fuel assembly without burnable absorbers, the power distribution changes only slightly over the depletion period. Furthermore, in the current PWR fuel assembly designs, any LLFP power peaking problem could be mitigated by employing burnable absorbers. Therefore, it can be said that a significant LLFP loading in the guide tube does not adversely affect the assembly power distribution. However, the LLFP loading in the guide tube would increase the heat flux from the guide tube to coolant due to additional gamma and neutron heating of the target and hence affect the departure from nucleate boiling ratio (DNBR) for the guide-tube channels. Although the LLFP loading in the guide tube is not expected to reduce the DNBR significantly since it reduces the neighboring pin powers as shown in Fig. 16, detailed thermalhydraulic analyses need to be performed to evaluate its effect on the minimum DNBR.

\section{TRANSMUTATION OF ${ }^{99}$ Tc AND ${ }^{129}$ I IN AN ACCELERATOR TRANSMUTATION OF WASTE SYSTEM}

Previous studies have shown that fast systems are more attractive transmuters because of the high neutron flux and the surplus neutrons that could be used for target transmutation. ${ }^{3,17}$ The high leakage fraction also pro- vides more flexibility for LLFP loading. To take advantage of higher capture cross sections for thermal neutrons, the neutron spectrum in LLFP target assemblies can also be moderated by employing metal hydrides such as $\mathrm{ZrH}_{2}$ and $\mathrm{CaH}_{2}$ (Refs. 3 through 8). These moderated target assemblies can be positioned in the inner core to take full advantage of the higher flux level of fast systems. (However, it requires additional design measures to reduce the local power peaking in nearby fuel pins caused by moderation in the target assembly.) Alternatively, neutron leakage from a fast reactor core can be utilized by locating LLFP target assemblies at the core periphery (e.g., in the reflector region).

To devise an optimal strategy for transmuting LLFPs in fast systems, trade studies were performed for an 840MW(thermal) sodium-cooled ATW system. Using metallic ${ }^{99} \mathrm{Tc}$ and $\mathrm{CaI}_{2}$ target forms and $\mathrm{ZrH}_{2}$ moderator, assembly design optimization studies were first done to maximize the LLFP transmutation performance and to mitigate the local power peaking problem in neighboring fuel assemblies caused by moderation in the target assembly. Then, using optimized LLFP target assembly designs, full-core depletion calculations were carried out for various target loading options including the loading of moderated target assemblies in the inner core and the reflector region and the homogeneous mixing of LLFP with fuel. For simultaneous loading of ${ }^{99} \mathrm{Tc}$ and ${ }^{129} \mathrm{I}$ target assemblies, further assembly design optimization was performed to equalize the ${ }^{99} \mathrm{Tc}$ and ${ }^{129} \mathrm{I}$ support ratios to the TRU support ratio for the burner design, ensuring that neither the long-lived TRUs nor LLFPs accumulate.

In Sec. IV.A, the methods and results of the assembly design optimization studies are presented. The LLFP loading option studies are discussed in Sec. IV.B along with the LLFP transmutation performances.

\section{IV.A. Long-Lived Fission Product Target Assembly Design Studies}

In the case of repeated recycle, the loss rate of LLFPs to the repository is basically determined by the discharge burnup (i.e., the fractional consumption at discharge) and the recovery factor per recycle pass. The loss rate monotonically decreases as the discharge burnup and recovery factor increase. Based on the ATW roadmap study, ${ }^{18}$ the (currently targeted) recovery factor for LLFP elements is $\sim 95 \%$, while it is over $99.9 \%$ for TRU elements. Considering this relatively low recovery factor, the discharge burnup of LLFP target assemblies should be maximized under various design and operation constraints.

For fixed total flux level and residence time, the LLFP discharge burnup is primarily determined by the effective one-group capture cross sections. To take advantage of higher LLFP capture cross sections for thermal neutrons, the LLFP target assemblies are generally moderated with metal hydrides such as $\mathrm{ZrH}_{2}$ and $\mathrm{CaH}_{2}$. 


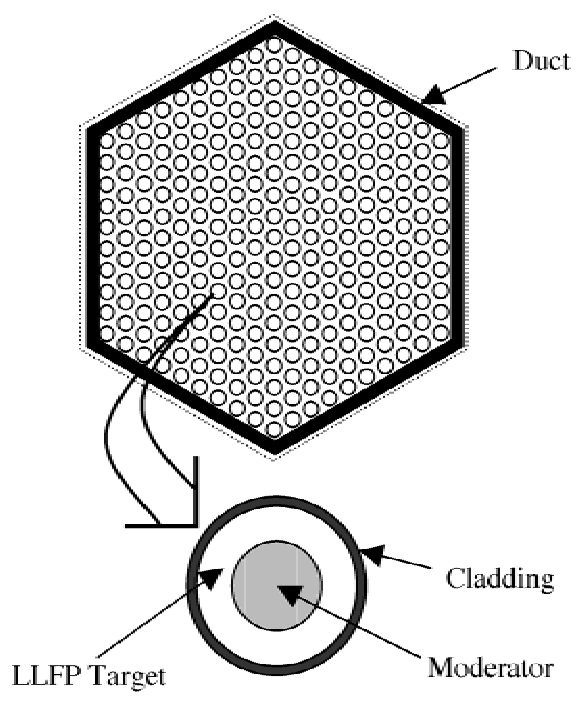

(a) Configuration of LLFP Assembly

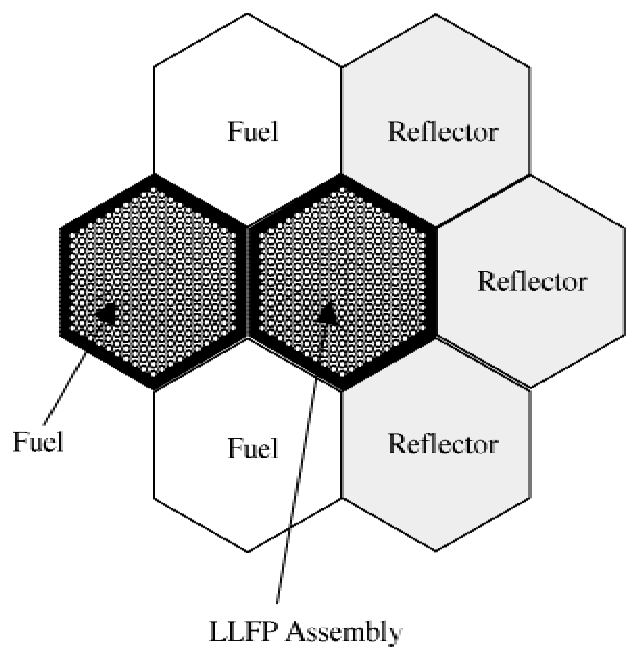

(b) VIM Model for Reflector Zone Loading

Fig. 17. (a) LLFP assembly configuration and (b) seven-assembly VIM computational model.

In a moderated LLFP target assembly, as the moderator loading fraction increases, the effective capture cross section (i.e., relative transmutation rate) increases due to the enhanced moderation and reduced self-shielding effects of LLFP isotopes. On the other hand, the increase of moderator loading fraction results in a reduced LLFP loading and hence a reduced absolute transmutation rate. As a result, it is expected that there exists an optimum moderator loading fraction that provides a good compromise between the goals of maximizing both the relative and absolute LLFP transmutation rates.

Spectral optimization studies were first performed for moderated ${ }^{99} \mathrm{Tc}$ and ${ }^{129} \mathrm{I}$ target assemblies. To account for the local heterogeneity effects, calculations were carried out with the VIM continuous Monte Carlo code. ${ }^{19}$ A variety of thermal neutron filter concepts were examined to reduce the local power peaking in neighboring fuel assemblies caused by target moderation.

\section{IV.A.1. Spectral Optimization}

For spectral optimization studies, a reference target assembly design was developed based on previous studies and preliminary analyses. In this design, target pins composed of annular technetium or iodine target material surrounding $\mathrm{ZrH}_{2}$ moderator within an HT-9 cladding are arranged in a triangular array inside a HT-9 hexagonal duct, as shown in Fig. 17a. Table VIII summarizes the assembly design parameters that were selected to be compatible with the ATW fuel assembly design. ${ }^{11}$ Metallic technetium was assumed for the ${ }^{99} \mathrm{Tc}$ target. As the iodine target material, a $\mathrm{CaI}_{2}$ compound was selected because of relatively high melting points of
$\mathrm{CaI}_{2}\left(783^{\circ} \mathrm{C}\right)$ and calcium $\left(842^{\circ} \mathrm{C}\right) .(\mathrm{NaI}$ is expected to melt when the sodium is liberated from iodine due to the transmutation, and this might be a problem for achieving a high discharge burnup.) Isotopic separation of ${ }^{129} \mathrm{I}$ was excluded, and an iodine vector of $77 \%{ }^{129} \mathrm{I}$ and $23 \%{ }^{127} \mathrm{I}$ (typical of PWR spent fuel) was used for the ${ }^{129} \mathrm{I}$ target.

For this reference assembly design, the optimum moderator fraction was first sought for each of the technetium and iodine target assemblies by adjusting the moderator volume fraction inside the cladding. For a given moderator volume fraction, two variations of target configuration were also investigated: cylindrical target surrounded by annular moderator and homogeneous mixture of LLFP target and moderator. To account for local heterogeneity effects, Monte Carlo calculations were performed with VIM. In order to examine the spectral and LLFP resonance self-shielding effects without introducing excessive computational

\section{TABLE VIII}

Design Parameters of Reference LLFP Target Assembly
Number of target pins per assembly

Pin diameter $(\mathrm{cm})$

Cladding thickness $(\mathrm{cm})$

$\mathrm{P} / \mathrm{D}$ ratio

Active length $(\mathrm{cm})$

Duct outside flat-to-flat $(\mathrm{cm})$

Duct wall thickness $(\mathrm{cm})$

Interassembly gap $(\mathrm{cm})$

Assembly pitch (cm)
271

0.74422

0.05588

1.1969

106.68

15.7099

0.3937

0.4318

16.1417 
TABLE IX

Moderator Effects on Effective Capture Cross Sections and Power Peaking Factor

\begin{tabular}{|c|c|c|c|c|c|}
\hline \multirow[b]{2}{*}{$\begin{array}{c}\mathrm{ZrH}_{2} \\
\text { Volume } \\
\text { Fraction }\end{array}$} & \multicolumn{2}{|c|}{${ }^{99}$ Tc Target } & \multicolumn{3}{|c|}{$\mathrm{CaI}_{2}$ Target } \\
\hline & $\begin{array}{c}\text { One-Group } \\
\text { Capture Cross } \\
\text { Section of }{ }^{99} \mathrm{Tc} \\
\text { (b) }\end{array}$ & $\begin{array}{l}\text { Power Peaking } \\
\text { Factor in } \\
\text { Fuel Assembly }\end{array}$ & $\begin{array}{l}\text { One-Group } \\
\text { Capture Cross } \\
\text { Section of }{ }^{127} \mathrm{I} \\
\text { (b) }\end{array}$ & $\begin{array}{l}\text { One-Group } \\
\text { Capture Cross } \\
\text { Section of }{ }^{129} \mathrm{I} \\
\text { (b) }\end{array}$ & $\begin{array}{l}\text { Power Peaking } \\
\text { Factor in } \\
\text { Fuel Assembly }\end{array}$ \\
\hline \multicolumn{6}{|c|}{ LLFP Target Loading in Reflector Region } \\
\hline $\begin{array}{l}0.0 \\
0.2 \\
0.4 \\
0.6 \\
0.8 \\
0.9\end{array}$ & $\begin{array}{l}0.43\left(0.43^{\mathrm{a}}\right) \\
0.78(0.49) \\
1.23(0.57) \\
1.94(0.70) \\
3.39(0.91) \\
4.99(1.12)\end{array}$ & $\begin{array}{l}1.09 \\
1.08 \\
1.28 \\
1.64 \\
2.22 \\
2.81\end{array}$ & $\begin{array}{l}0.71\left(0.71^{\mathrm{a}}\right) \\
2.19(0.83) \\
3.00(0.99) \\
3.70(1.24) \\
4.52(1.71) \\
5.18(2.12)\end{array}$ & $\begin{array}{l}0.37\left(0.37^{\mathrm{a}}\right) \\
1.20(0.43) \\
2.11(0.51) \\
3.31(0.61) \\
4.98(0.79) \\
6.11(0.92)\end{array}$ & $\begin{array}{l}1.07 \\
1.54 \\
2.14 \\
2.74 \\
3.32 \\
3.67\end{array}$ \\
\hline \multicolumn{6}{|c|}{ LLFP Target Loading in Inner Core } \\
\hline 0.6 & 1.84 & 1.87 & 3.54 & 3.10 & 3.44 \\
\hline
\end{tabular}

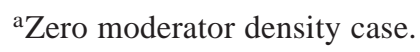

burden, two seven-assembly models were developed for target assembly loadings in the inner core and reflector regions; an LLFP target assembly is surrounded by six fuel assemblies in the model for the inner core loading, but it is surrounded by three fuel assemblies and three HT-9 reflector assemblies in the model for the reflector region loading as shown in Fig. 17b. The fuel assembly was loaded with a Zr-based metal dispersion fuel, and the TRU isotopic vector of LWR spent nuclear fuel was used. In both models, one of the fuel assemblies was evaluated in detail to assess the local power peaking due to the thermal neutron leakage from the LLFP assembly. With the reflective boundary conditions imposed, the VIM calculations were performed with the ENDF/B-V crosssection library at $300 \mathrm{~K}$.

Using these seven-assembly computational models, Monte Carlo VIM calculations were carried out for various moderator volume fractions by adjusting the target thickness. In order to separate out the spectrum softening effects, the VIM calculation for each target thickness was repeated by setting moderator density to zero. The results are summarized in Table IX and in Figs. 18, 19, and 20. Table IX shows the effective one-group capture cross sections of ${ }^{99} \mathrm{Tc},{ }^{127} \mathrm{I}$, and ${ }^{129} \mathrm{I}$ along with the power peaking factor in the fuel assembly. The results show that the capture cross sections of the three isotopes monotonically increase as the moderator volume increases. This is due to the reduced resonance self-shielding and the softened neutron spectrum. Even in the zero moderator density case, the capture cross section increases as the target thickness decreases due to the reduced spatial self-shielding effects. Compared to ${ }^{99} \mathrm{Tc},{ }^{129} \mathrm{I}$ shows a substantially larger increase of the effective capture cross section because of its higher thermal capture cross section. The increase of the ${ }^{99} \mathrm{Tc}$ cross section is largely due to the reduced self-shielding of the large resonance peak at $5.6 \mathrm{eV}$. (The resonance integral is $\sim 340 \mathrm{~b}$ for ${ }^{99} \mathrm{Tc}$, while it is $\sim 36 \mathrm{~b}$ for ${ }^{129} \mathrm{I}$.) These energy effects are illustrated in Fig. 18, which shows the normalized capture reaction rate distributions over a 27 -group structure for a $60 \%$ moderator volume. The thermal group reaction rate is much larger than those of other groups in the ${ }^{129} \mathrm{I}$ target. For the ${ }^{99} \mathrm{Tc}$ target, the thermal reaction rate is

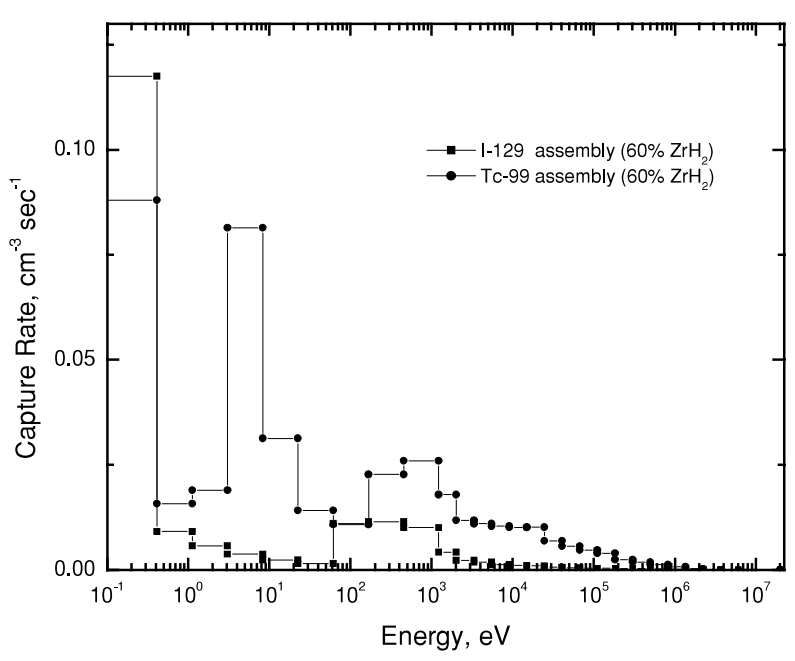

Fig. 18. Groupwise capture reaction rate in LLFP assemblies with a $60 \%$ moderator volume. 


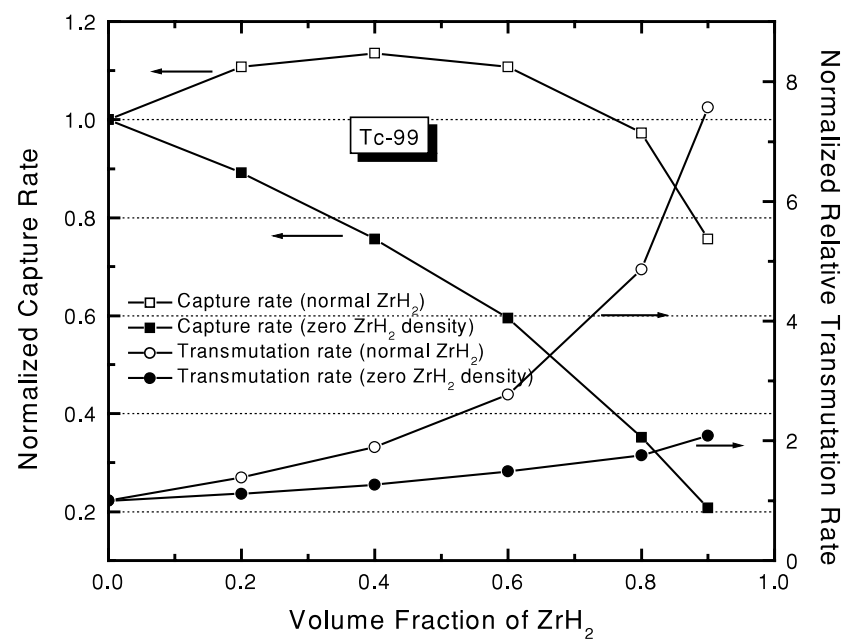

Fig. 19. Transmutation rates of ${ }^{99} \mathrm{Tc}$ versus moderator volume fraction (reflector loading case).

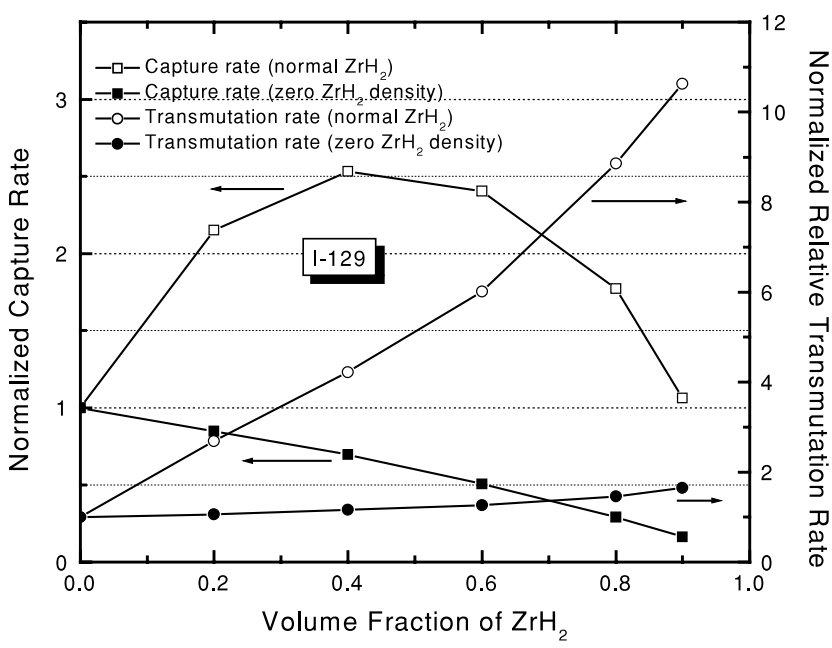

Fig. 20. Transmutation rates of ${ }^{129}$ I versus moderator volume fraction (reflector loading case).

slightly larger than that of the 24th group, which contains a large resonance. In the case of inner core loading, the capture cross sections are slightly reduced relative to the reflector loading case due to harder neutron spectrum.

As shown in Table IX, the $\mathrm{ZrH}_{2}$ moderator in the LLFP target assembly induces a large local power peaking in the adjacent fuel assemblies. The power peaking problem is more pronounced in the iodine target assembly case. This is mainly due to the larger mean-free-path of thermal neutrons in $\mathrm{CaI}_{2}$ than in technetium; the thermal neutron mean path is about three times larger in $\mathrm{CaI}_{2}$ than in technetium since the iodine atom density in the $\mathrm{CaI}_{2}$ target is $\sim 3.8$ times smaller than the ${ }^{99} \mathrm{Tc}$ atom density in the metallic technetium target. The extra neu- tron moderation by the calcium nuclides also contributes. Compared to the reflector loading case, the power peaking of the inner core loading case is considerably higher for the ${ }^{129}$ I target assembly and marginally higher for the ${ }^{99} \mathrm{Tc}$ target assembly.

In Figs. 19 and 20, the capture reaction rates and relative transmutation rates normalized to the unmoderated case are plotted as a function of the moderator volume fraction. It can be observed that a moderator fraction in the vicinity of 0.4 provides the maximum capture rate, that is, the maximum absolute transmutation rate (LLFP mass transmuted per unit time). With this optimal moderator volume fraction, the capture rate is increased by $\sim 15 \%$ for ${ }^{99} \mathrm{Tc}$ and by $\sim 150 \%$ for ${ }^{129} \mathrm{I}$ relative to the unmoderated case. The ${ }^{129} \mathrm{I}$ capture rate is increased much more significantly by moderation because the capture cross section of ${ }^{129} \mathrm{I}$ is greatly enhanced by the $\mathrm{ZrH}_{2}$ moderator due to its higher thermal cross section. Comparing the capture rates of the normal and zero moderator cases, most of these capture rate increases can be ascribed to the moderator. These results show that a desired absolute transmutation rate can be achieved with a much smaller LLFP loading by employing moderator. For a fixed amount of LLFP loading per target assembly, the maximum transmutation performance is achieved by arranging the LLFP target pins in such a way that the moderator volume is maximized. This suggests that total volume for the target and moderator should be maximized subject to assembly thermal design constraints.

Figures 19 and 20 also show that the relative transmutation rate (fraction of LLFP transmuted per a unit time) increases monotonically with the moderator volume fraction. As a result, the LLFP loading needs to be minimized in order to obtain the maximum target discharge burnup, which is desired to reduce the LLFP loss to the repository. On the other hand, in order to maximize the LLFP incineration, the moderator volume fraction needs to be selected in the vicinity of 0.4 as discussed above; in the region of moderator volume fraction above 0.4 , the absolute transmutation rate decreases monotonically with the moderator volume fraction. Furthermore, the power peaking in nearby fuel assemblies also increases monotonically as the moderator volume fraction increases. Considering these three conflicting performance indices, it is believed that a moderator volume fraction of 0.6 to 0.7 is a good compromise.

For the $60 \%$ moderator volume fraction, two variations of target configuration were also investigated: (a) central cylindrical target surrounded by annular moderator and (b) homogeneous mixture of LLFP target and moderator. The effective one-group capture cross sections are compared in Table X. In terms of the relative transmutation rate, the homogeneous mixture provides the best performance (due to the minimized self-shielding effect), increasing the rate by $\sim 5 \%$, and the central cylindrical target gives the worst performance, but the 
TABLE $X$

Effective Capture Cross Sections of Different Target Configurations*

\begin{tabular}{|c|c|c|c|}
\hline \multirow[b]{2}{*}{ Target Configuration } & \multirow{2}{*}{$\begin{array}{c}{ }^{99} \mathrm{Tc} \text { Target } \\
\text { One-Group } \\
\text { Capture Cross } \\
\text { Section of }{ }^{99} \mathrm{Tc} \\
\text { (b) }\end{array}$} & \multicolumn{2}{|c|}{$\mathrm{CaI}_{2}$ Target } \\
\hline & & $\begin{array}{l}\text { One-Group } \\
\text { Capture Cross } \\
\text { Section of }{ }^{127} \mathrm{I} \\
\text { (b) }\end{array}$ & $\begin{array}{l}\text { One-Group } \\
\text { Capture Cross } \\
\text { Section of }{ }^{129} \mathrm{I} \\
\quad \text { (b) }\end{array}$ \\
\hline $\begin{array}{l}\text { Annular LLFP target surrounding cylindrical moderator (reference) } \\
\text { Central cylindrical LLFP target surrounded by annular moderator } \\
\text { Homogeneous mixture of LLFP and moderator }\end{array}$ & $\begin{array}{l}1.94 \\
1.87 \\
2.01\end{array}$ & $\begin{array}{l}3.70 \\
3.46 \\
3.93\end{array}$ & $\begin{array}{l}3.31 \\
3.30 \\
3.37\end{array}$ \\
\hline
\end{tabular}

$*$ Moderator volume fraction $=60 \%$.

differences are small. Furthermore, the power peaking problem is exacerbated in the homogeneous mixture and the annular moderator options. In addition, it is not clear at this moment whether the homogeneous mixing concept is technically feasible from the fabrication/irradiation point of view.

For the same moderator volume fraction in the LLFP target assembly, these target designs (Fig. 17a) generally provide larger LLFP capture cross sections due to the reduced self-shielding effects, compared to previous studies $^{3,8}$ where distinct LLFP target and moderator pins are employed either with a uniform pin diameter or with an irregular pin diameter (larger moderator pin diameter). Therefore, in terms of the effective LLFP capture cross sections, the LLFP target assembly based on the annular target concept could be considered as a near-optimal design. However, the material compatibility between LLFP targets and $\mathrm{ZrH}_{2}$ moderator needs to be confirmed, although no serious compatibility issue is anticipated.

\section{IV.A.2. Thermal Neutron Filter}

As discussed in Sec. IV.A.1, the moderated LLFP target assembly substantially enhances the LLFP transmutation rates. On the other hand, the moderation in the target assembly also results in an increased local power peaking in the nearby fuel assemblies. To mitigate the power peaking, a thicker duct wall has been proposed. ${ }^{4}$ However, this option is not attractive from the neutron utilization viewpoint. To reduce the thermal neutron leakage from the LLFP assembly to the neighboring fuel assemblies, a ${ }^{99} \mathrm{Tc}$ plate could be placed between the duct wall and the target pins. ${ }^{8}$ This latter scheme has an advantage in that the leaking thermal neutrons can be utilized to transmute ${ }^{99} \mathrm{Tc}$ in the thermal neutron filter, although its transmutation rate is relatively lower compared to the interior targets. In this work, a variant of the latter option was examined by replacing the target pins in the outer rings with unmoderated ${ }^{99} \mathrm{Tc}$ pins along the boundary between the LLFP and fuel assemblies. For the
${ }^{129}$ I target assembly, the $\mathrm{CaI}_{2}$ target pins in two outer rings were replaced with cylindrical ${ }^{99} \mathrm{Tc}$ target pins. In the case of the ${ }^{99} \mathrm{Tc}$ target assembly, the internal moderator was removed from the target pins in the outermost ring. Figure 21 shows the configurations of the ${ }^{99} \mathrm{Tc}$ and ${ }^{129} \mathrm{I}$ target assemblies with thermal neutron filters.

Using the seven-assembly VIM model for the reflector loading case, the effects of the ${ }^{99} \mathrm{Tc}$ thermal neutron filter were investigated for ${ }^{99} \mathrm{Tc}$ and ${ }^{129} \mathrm{I}$ target assemblies. For comparison, each calculation was repeated by replacing the ${ }^{99} \mathrm{Tc}$ pins with HT-9 pins. The resulting local power peaking factors are presented in Table XI. By employing the unmoderated ${ }^{99} \mathrm{Tc}$ pins as the thermal neutron filter, the initial local power peaking factor was reduced from 1.64 to 1.43 for the ${ }^{99} \mathrm{Tc}$ target assembly and from 2.74 to 1.40 for the ${ }^{129} \mathrm{I}$ target assembly. On the other hand, the effective capture cross sections were slightly reduced because of reduced moderation, as expected. The results also show that HT-9 pins are not as efficient as ${ }^{99} \mathrm{Tc}$ pins as the thermal neutron filter, especially for the ${ }^{129}$ I target assembly; for sufficient filtering of thermal neutrons, more ${ }^{129} \mathrm{I}$ target pins would need to

\section{TABLE XI}

Local Power Peaking of Fuel Assembly Neighboring to LLFP Target Assembly with Thermal Neutron Filter*

\begin{tabular}{|c|l|c|c|}
\hline \multirow{2}{*}{$\begin{array}{c}\text { LLFP } \\
\text { Burnup } \\
\text { (at.\%) }\end{array}$} & \multirow{2}{*}{$\begin{array}{c}\text { Thermal } \\
\text { Neutron } \\
\text { Filter }\end{array}$} & \multicolumn{2}{|c|}{$\begin{array}{c}\text { Power Peaking in Neighboring } \\
\text { Fuel Assembly }\end{array}$} \\
\cline { 3 - 4 } 0 & ${ }^{99}$ Tc Target & CaI $_{2}$ Target \\
\hline 0 & No filter & 1.64 & 2.74 \\
& HT-9 & 1.49 & 2.04 \\
& ${ }^{99} \mathrm{Tc}$ & 1.43 & 1.40 \\
40 & ${ }^{99} \mathrm{Tc}$ & 1.46 & 1.55 \\
\hline
\end{tabular}

*Moderator volume fraction $=60 \%$. 


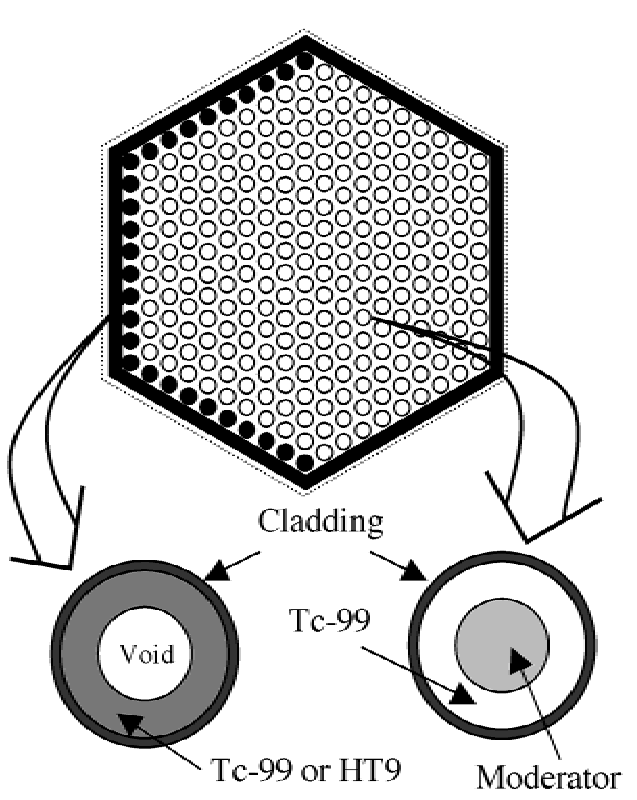

(a) Tc-99 Target Assembly

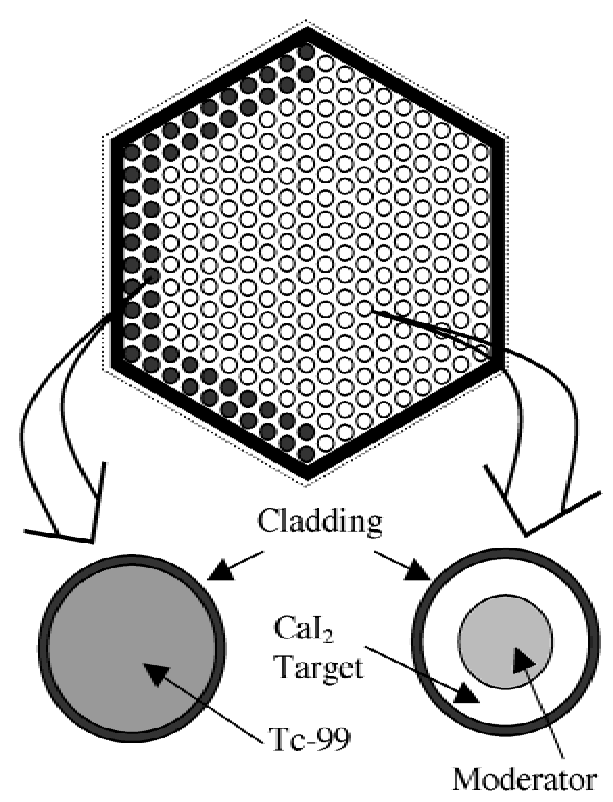

(b) I-129 Target Assembly

Fig. 21. Thermal neutron filters for ${ }^{99} \mathrm{Tc}$ and ${ }^{129} \mathrm{I}$ target assemblies.

be replaced by the HT-9 pins, penalizing the ${ }^{129}$ I transmutation performance.

By neutron capture, technetium and iodine isotopes are converted mainly into ${ }^{100} \mathrm{Ru}$ and ${ }^{130} \mathrm{Xe}$, respectively. Since the capture cross sections of these product isotopes are significantly smaller than those of target isotopes, the power peaking is likely to increase with target burnup. Therefore, the power peaking factors were estimated for a 40 at. $\%$ burnup of ${ }^{99} \mathrm{Tc},{ }^{127} \mathrm{I}$, and ${ }^{129} \mathrm{I}$. To get a conservative estimate, it was assumed that ${ }^{127} \mathrm{I}$ and ${ }^{129} \mathrm{I}$ are converted into void and ${ }^{99} \mathrm{Tc}$ is converted into ${ }^{101} \mathrm{Ru}$, which has a slightly smaller thermal capture cross section than ${ }^{100} \mathrm{Ru}$. As shown in Table XI, the high LLFP burnup increases the power peaking slightly. However, this power peaking increase is considered tolerable based on the conservative peaking factor evaluation.

The power peaking factors presented in Table XI provide conservative estimates for the LLFP assembly loading in the reflector region because a reflective boundary condition was imposed. In the actual situation, a steep flux gradient exists across the fuel, target, and reflector assemblies that would likely reduce the power peaking in the adjacent fuel assembly. In addition, the LWR spentfuel TRU vector used in these calculations has a high ${ }^{239} \mathrm{Pu}$ fraction $(>50 \%)$, enhancing the thermal neutroninduced power peak; in an equilibrium core, the ${ }^{239} \mathrm{Pu}$ fraction would be significantly depressed, helping reduce the peaking factor. With appropriate coolant flow allocation, the power peaking factors shown in Table XI can be tolerated since the power densities in the peripheral fuel assemblies are much lower than the core aver- age power density. On the other hand, the power peaking needs to be reduced further when LLFP target assemblies are loaded in the inner core.

\section{IV.B. Long-Lived Fission Product \\ Transmutation Performance of an Accelerator Transmutation of Waste System}

Using the LLFP target assembly designs discussed in Sec. IV.A, the ${ }^{99} \mathrm{Tc}$ and ${ }^{129} \mathrm{I}$ transmutation capability of a sodium-cooled ATW system ${ }^{11}$ was investigated by performing full-core depletion calculations for various target loading options including the moderated target assembly loading in the inner core and in reflector regions and a homogeneous mixing of LLFP with fuel. ATW system design parameters are based on the studies in Ref. 11. For simultaneous loading of ${ }^{99} \mathrm{Tc}$ and ${ }^{129} \mathrm{I}$ target assemblies, further assembly design optimization was performed such that all ${ }^{99} \mathrm{Tc}$ and ${ }^{129} \mathrm{I}$ produced in conventional PWRs supported by an ATW system can be incinerated in the same ATW system without being accumulated (i.e., to equalize the ${ }^{99} \mathrm{Tc}$ and ${ }^{129} \mathrm{I}$ support ratios to the TRU support ratio).

The LLFP transmutation performances were evaluated by REBUS-3 (Ref. 20) equilibrium cycle analyses. Region-dependent 27-group cross sections were generated using the $\mathrm{MC}^{2}-2 \operatorname{code}^{21}$ based on ENDF/B-V data. Since $\mathrm{MC}^{2}-2$ is not designed to generate the thermal group data, the thermal group $(E<0.414 \mathrm{eV})$ cross sections for the moderated LLFP target assemblies were estimated for the seven-assembly models shown in Fig. 17 using the VIM code based on the ENDF/B-V library. 
Flux calculations were performed with triangular- $Z$ models using the finite difference diffusion theory option. The core region is modeled with two "enrichment" zones with a higher TRU/Zr ratio in the outer zone. Fuel regions are modeled with batch-averaged compositions for a seven-batch fuel management scheme without fuel shuffling. Target regions are also modeled with batch-averaged compositions over the target assembly lifetime constrained by the peak fast fluence limit on the cladding.

\section{IV.B.1. Long-Lived Fission Product Transmutation Objectives}

The main design objective of the sodium-cooled ATW system is to consume TRU as efficiently as possible. To limit the burnup reactivity swing, a half-year cycle (135 EFPD) is adopted. The charged fuel contains the TRUs recovered via recycle from the discharged ATW fuel, supplemented by LWR-discharge TRU to make up for the TRU consumed by fission. During recycle and refabrication, it is assumed that all TRU discharged are recovered and $5 \%$ of the rare-earth FPs are carried over with the recycled TRU. A schematic configuration of the ATW core is shown in Fig. 22. The detailed core design characteristics of the sodium-cooled ATW can be found in Ref. 11.

Unless special systems dedicated to the LLFP incineration are employed, simultaneous transmutation of both TRU and LLFP (in the appropriate proportion) appears to be highly desirable. In this respect, the LLFP transmutation goal was defined as achieving ${ }^{99} \mathrm{Tc}$ and ${ }^{129} \mathrm{I}$ support ratios equal to the TRU support ratio. In order to estimate these support ratios, the ${ }^{99} \mathrm{Tc},{ }^{129} \mathrm{I}$, and TRU production rates in a typical PWR and the ATW system

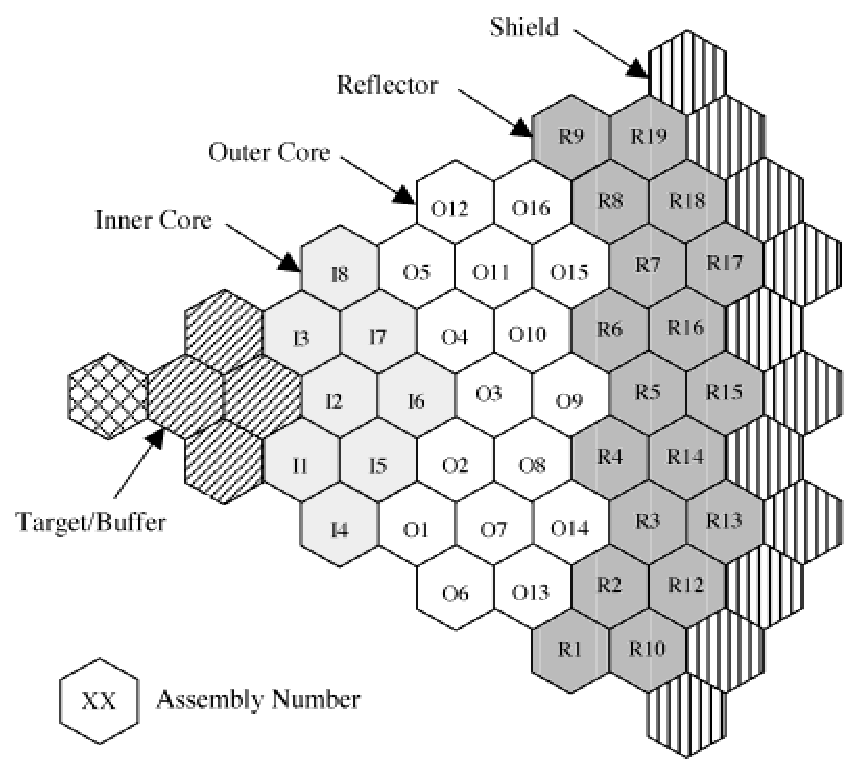

Fig. 22. Planar layout of 840-MW(thermal) sodiumcooled ATW core (one-sixth core). were calculated using the ORIGEN2 code. $\mathrm{A} \mathrm{UO}_{2}$ fuel composition (typical of a PWR) with $3.2 \mathrm{wt} \%{ }^{235} \mathrm{U}$ enrichment was depleted up to $33 \mathrm{GWd} /$ tonne $\mathrm{U}$ burnup, and a TRU fuel composition was depleted up to 270 $\mathrm{GWd} /$ tonne HM burnup, which is the typical fuel discharge burnup of the ATW system. The results are summarized in Table XII; assuming that the PWR and ATW system capacity factors are 80 and $74 \%$, respectively, the TRU support ratio of the $840-\mathrm{MW}$ (thermal) ATW system is estimated to be 3.2. Therefore, in order to achieve the same support ratios while accounting for LLFP production in the ATW system, $22.8 \mathrm{~kg}$ of ${ }^{99} \mathrm{Tc}$ and $5.6 \mathrm{~kg}$ of ${ }^{129} \mathrm{I}$ need to be consumed per year (i.e., $11.4 \mathrm{~kg}$ of ${ }^{99} \mathrm{Tc}$ and $2.8 \mathrm{~kg}$ of ${ }^{129} \mathrm{I}$ per cycle) in the target assemblies.

\section{IV.B.2. Heterogeneous Transmutation}

The heterogeneous transmutation approach was first investigated by focusing on ${ }^{99} \mathrm{Tc}$ transmutation. Two loading options were analyzed. In the first option, 12 moderated ${ }^{99} \mathrm{Tc}$ target assemblies were loaded into positions R4 and R6 of the reflector (see Fig. 22) and their symmetric counterparts. In the second option, six target assemblies were loaded in the $\mathrm{O} 3$ position within the core and its symmetric counterparts.

In the reflector loading case, the R4 and R6 positions were selected because they have higher flux levels than the other reflector positions. The moderated ${ }^{99} \mathrm{Tc}$ target assembly with the ${ }^{99} \mathrm{Tc}$ thermal neutron filter was used; the 28 target pins at the interface between the fuel and target assemblies are composed of the annular ${ }^{99} \mathrm{Tc}$ target without moderator, and the other target pins are composed of the annular ${ }^{99} \mathrm{Tc}$ target and $\mathrm{ZrH}_{2}$ moderator with a $60 \%$ moderator volume fraction. The number of target assemblies was determined such that the ${ }^{99} \mathrm{Tc}$ transmutation rate in the target assemblies is roughly $11 \mathrm{~kg} /$ cycle.

In the case of the core interior loading, the location of the target assembly should be determined in such a way that a high transmutation rate can be achieved while preserving the central source multiplication. For example, although the target assembly loaded in the innermost fuel ring near the spallation target would produce the highest ${ }^{99} \mathrm{Tc}$ transmutation rate due to the flux level

TABLE XII

Production Rates of TRU, ${ }^{99} \mathrm{Tc}$, and ${ }^{129} \mathrm{I}$ in PWR and ATW Systems

\begin{tabular}{|c|c|c|c|}
\hline \multirow[b]{2}{*}{ Fuel Type } & \multicolumn{3}{|c|}{$\begin{array}{c}\text { Production Rate } \\
{[\mathrm{kg} / \mathrm{GW}(\text { thermal }) \cdot \mathrm{yr}]}\end{array}$} \\
\hline & TRU & ${ }^{99} \mathrm{Tc}$ & ${ }^{129} \mathrm{I}$ \\
\hline PWR (33 GWd/tonne U burnup) & 107.9 & 8.6 & 2.0 \\
\hline ATW (270 GWd/tonne HM burnup) & -369.1 & 7.1 & 2.2 \\
\hline
\end{tabular}


being highest, it reduces the source multiplication efficiency significantly by directly absorbing the source neutrons. In this study, the $\mathrm{O} 3$ location was selected as a compromise between the flux level and the negative impact on the source multiplication. To reduce the local power peaking, solid cylindrical ${ }^{99} \mathrm{Tc}$ pins were loaded in the two outermost rings of the target assembly, and the moderator volume fraction of the internal target pins was increased to $70 \%$ to balance the transmutation rate.

The lifetime of the moderated target assembly would likely be constrained by the fast neutron irradiation damage to the cladding (fast fluence limit) and/or irradiation damage to the moderator. Based on the worldwide operation histories of TRIGA reactors in which $\mathrm{ZrH}_{2}$ moderator is incorporated with enriched uranium metal in the fuel elements, it is expected that the $\mathrm{ZrH}_{2}$ disintegration would not be a main obstacle to achieving this irradiation time. Thus, the irradiation time of the ${ }^{99} \mathrm{Tc}$ target assembly was constrained by the fast fluence limit of HT-9 cladding $\left(4 \times 10^{23} \mathrm{n} / \mathrm{cm}^{2}\right)$. From the REBUS-3 equilibrium cycle analysis, it was estimated that the ${ }^{99} \mathrm{Tc}$ target assembly could be irradiated for 14 consecutive fuel cycles $(5.2 \mathrm{yr})$ before the fast fluence limit is reached. Thus, the target burnup was determined by the REBUS-3 equilibrium cycle analyses with a target residence time of 14 cycles and seven- and eight-batch fuel management schemes for the inner and outer zones, respectively.

The resulting ${ }^{99} \mathrm{Tc}$ transmutation rates and selected core performance parameters are presented in Table XIII. It can be observed that the two ${ }^{99} \mathrm{Tc}$ transmutation options could meet the required ${ }^{99} \mathrm{Tc}$ support ratio by slightly increasing the ${ }^{99} \mathrm{Tc}$ loading. The in-core burning of ${ }^{99} \mathrm{Tc}$ provides a $25 \%$ higher relative transmutation rate than the reflector loading case, due to the higher flux level.
Although the flux level is almost doubled in the in-core burning case, the increase in the transmutation rate is not proportionally high. This is attributed to the fact that the effective capture cross section of ${ }^{99} \mathrm{Tc}$ is significantly smaller in the in-core case due to the larger loading per assembly and the harder neutron spectrum than in the reflector loading. For the in-core loading, the 46 at.\% discharge burnup might be a maximum value due to the fluence limit. However, in the case of the reflector loading, the target discharge burnup could be significantly increased with a longer irradiation time: The peak fast fluence is only $\sim 62 \%$ of the limit.

As shown in Table XIII, the in-core burning option requires a slightly higher TRU fuel inventory than the reflector loading case and hence results in $4 \%$ lower fuel discharge burnup, although the absolute ${ }^{99} \mathrm{Tc}$ transmutation rate (kilogram/cycle) is comparable in both cases. This is due to the relatively large neutron leakage in the ATW core. The in-core burning requires additional neutrons to compensate the neutrons absorbed by the ${ }^{99} \mathrm{Tc}$ target, whereas the ${ }^{99} \mathrm{Tc}$ target in the reflector is mainly transmuted by the neutrons leaking out of the core. This indicates that ${ }^{99} \mathrm{Tc}$ placement in the reflector is preferable to the in-core burning from the neutron economy point of view.

As discussed above, the local power peaking factor of 1.43 for the reflector loading case is considered to be acceptable since the power densities in the core peripheral fuel assemblies are much lower than the core average power density $\left(240 \mathrm{~W} / \mathrm{cm}^{3}\right)$. However, the in-core burning option increases the average core power density to $252 \mathrm{~W} / \mathrm{cm}^{3}$ because of the reduced number of fuel assemblies. This increased power density makes the local power peaking problem worse, and hence the local

TABLE XIII

Transmutation Performance Parameters of Heterogeneous ${ }^{99} \mathrm{Tc}$ Loading Options in Equilibrium Cycle ATW Core

\begin{tabular}{|c|c|c|}
\hline \multirow[b]{2}{*}{ Parameter } & \multicolumn{2}{|c|}{ Tc Assembly Location } \\
\hline & Core & Reflector \\
\hline 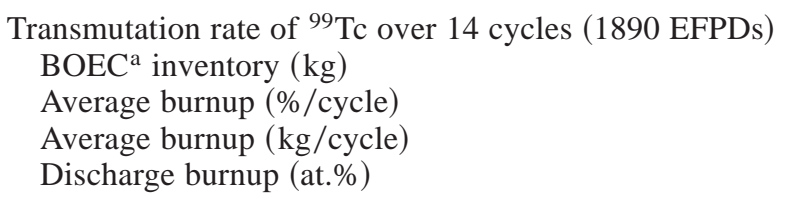 & $\begin{array}{c}232.2 \\
3.26 \\
9.91 \\
45.6\end{array}$ & $\begin{array}{c}316.7 \\
2.61 \\
10.1 \\
36.5\end{array}$ \\
\hline 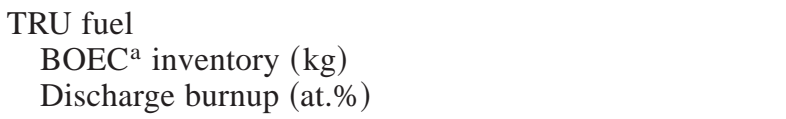 & $\begin{array}{r}3054 \\
25.8\end{array}$ & $\begin{array}{r}2911 \\
26.9\end{array}$ \\
\hline $\begin{array}{l}\text { Local power peaking in seven-assembly model } \\
\text { Initial total flux in Tc target assembly }\left(\mathrm{n} / \mathrm{cm}^{2} \cdot \mathrm{s}\right) \\
\text { Peak discharge fast fluence in Tc assembly }\left(\mathrm{n} / \mathrm{cm}^{2}\right)\end{array}$ & $\begin{array}{c}1.39 \\
2.66 \mathrm{E}+15^{\mathrm{b}} \\
3.95 \mathrm{E}+23\end{array}$ & $\begin{array}{c}1.43 \\
1.37 \mathrm{E}+15 \\
2.46 \mathrm{E}+23\end{array}$ \\
\hline
\end{tabular}

${ }^{\mathrm{a} B O E C}=$ beginning-of-equilibrium-cycle.

${ }^{b}$ Read as $2.66 \times 10^{15}$. 
power peaking factor of 1.39 might not be acceptable. In order to reduce the power peaking, the thermal neutron leakage should be further reduced by decreasing the moderator volume and/or by increasing the number of peripheral filter pins. This modification would reduce the ${ }^{99} \mathrm{Tc}$ transmutation rate. Alternatively, the power peaking problem can be mitigated by reducing the TRU fuel enrichment in the adjacent fuel assemblies. However, this makes the core design and fuel management more complicated and increases the fuel fabrication cost.

The in-core LLFP transmutation has another potential problem from the safety point of view. In general, the reactivity effect of the LLFP target assembly is quite large when it is loaded in the middle of the core. As a result, an accident involving the loss of target materials could inject a large amount of reactivity into the core. Meanwhile, the corresponding reactivity of an LLFP assembly in the reflector zone is much smaller, compared with the in-core case. Consequently, any melting or ejection of an LLFP assembly from the reflector would be more benign.

Transmutation of LLFP in the ATW reflector displaces steel reflectors, while still providing adequate shielding. Therefore, the LLFP burning in the reflector reduces the generation of steel activation products. This is viewed as another advantage of reflector loading over in-core target placement.

\section{IV.B.3. Homogeneous Transmutation}

Another option for ${ }^{99} \mathrm{Tc}$ transmutation is direct mixing of ${ }^{99} \mathrm{Tc}$ with fuel. In this so-called homogeneous transmutation option, the high neutron flux in the fast reactor core can effectively be used without adverse selfshielding of ${ }^{99} \mathrm{Tc}$. One disadvantage of this approach is that the effective capture cross section is small due to the hard neutron spectrum. This option is not considered for iodine transmutation because of the xenon gas production, which could adversely affect the fuel performance.

As shown in Fig. 22, the sodium-cooled ATW core employs two TRU enrichment zones (low-enrichment inner zone and high-enrichment outer zone) to flatten the radial power distribution. Thus, if ${ }^{99} \mathrm{Tc}$ is loaded uniformly in the whole core, the required outer zone TRU enrichment would become too high. On the other hand, since the flux level of the ATW core is significantly higher in the inner zone than in the outer region, the ${ }^{99} \mathrm{Tc}$ would be more effectively burned in the inner zone; however, too much loading of ${ }^{99} \mathrm{Tc}$ in the inner zone may seriously reduce the multiplication efficiency of the external neutron source. Taking these factors into account, ${ }^{99} \mathrm{Tc}$ was loaded in such a way that the loading density of ${ }^{99} \mathrm{Tc}$ in the inner core is about twice that of the outer zone. In addition, ${ }^{99} \mathrm{Tc}$ was not loaded in the outermost fuel ring to enhance the burnup of ${ }^{99} \mathrm{Tc}$ and flatten the radial power distribution.

The ${ }^{99} \mathrm{Tc}$ transmutation performance of ATW for the homogeneous loading option was assessed by the
REBUS-3 equilibrium cycle analyses. Seven- and eightbatch fuel management schemes were utilized for the inner and outer zones, respectively. The results are summarized in Table XIV. As discussed before, the ${ }^{99} \mathrm{Tc}$ discharge burnup decreases as the ${ }^{99} \mathrm{Tc}$ loading increases, while the absolute transmutation rate (kilogram/cycle) increases with the ${ }^{99} \mathrm{Tc}$ loading. By comparing Tables XIII and XIV, it can be observed that for the same ${ }^{99} \mathrm{Tc}$ loading, the homogeneous loading option results in transmutation performance inferior to the heterogeneous loading options. The significantly lower ${ }^{99} \mathrm{Tc}$ discharge burnup is mainly due to the short irradiation time: 945 days in the inner core and 1080 days in the outer core. The lower cycle burnup is caused by the small capture cross section resulting from the hard neutron spectrum. The homogeneous loading option also requires a larger TRU fuel inventory than the heterogeneous loading in the reflector for a comparable ${ }^{99} \mathrm{Tc}$ transmutation rate.

\section{IV.B.4. Simultaneous Transmutation of ${ }^{99} \mathrm{Tc}$ and ${ }^{129} \mathrm{I}$}

In order to achieve support ratios for ${ }^{99} \mathrm{Tc}$ and ${ }^{129} \mathrm{I}$ equal to the TRU support ratio, the simultaneous transmutation of both ${ }^{99} \mathrm{Tc}$ and ${ }^{129} \mathrm{I}$ in the ATW core was studied. Based on the above loading option studies, it was decided to load moderated LLFP target assemblies in the reflector region. The moderated LLFP target assembly designs with the ${ }^{99} \mathrm{Tc}$ thermal neutron filter discussed in Sec. IV.A. 2 were used. The reflector positions that have relatively higher flux levels were selected for LLFP assembly loading. Twelve ${ }^{129}$ I target assemblies were loaded into the R4 and R6 positions and their symmetric counterparts (see Fig. 22), which have the highest flux levels in the reflector region, and twelve ${ }^{99} \mathrm{Tc}$ target assemblies were loaded into the R2 and R8 positions and their symmetric counterparts, which have the next highest flux levels.

The resulting LLFP transmutation rates showed that the ${ }^{99} \mathrm{Tc}$ transmutation rate is sufficient to meet the support ratio goal, but the ${ }^{129} \mathrm{I}$ transmutation rate is not. Therefore,

\section{TABLE XIV}

Transmutation Performance Parameters of Homogeneous

${ }^{99}$ Tc Loading Option in Equilibrium Cycle ATW Core

\begin{tabular}{|c|c|c|c|c|c|}
\hline \multirow[b]{2}{*}{$\begin{array}{c}\text { Initial } \\
{ }^{99} \mathrm{Tc} \\
\text { Inventory } \\
(\mathrm{kg})\end{array}$} & \multicolumn{3}{|c|}{${ }^{99} \mathrm{Tc}$ Transmutation Rate } & \multirow[b]{2}{*}{$\begin{array}{c}\text { Initial } \\
\text { Fuel } \\
\text { Inventory } \\
(\mathrm{kg})\end{array}$} & \multirow{2}{*}{$\begin{array}{c}\text { Fuel } \\
\text { Discharge } \\
\text { Burnup } \\
\text { (at.\%) }\end{array}$} \\
\hline & $\% /$ cycle & $\mathrm{kg} /$ cycle & $\begin{array}{c}\text { Discharge } \\
\text { Burnup } \\
\text { (at.\%) }\end{array}$ & & \\
\hline 0 & - & - & - & 2744 & 28.3 \\
\hline 45 & 2.90 & 1.30 & 19.8 & 2775 & 28.0 \\
\hline 89 & 2.81 & 2.50 & 19.1 & 2813 & 27.7 \\
\hline 176 & 2.68 & 4.72 & 18.2 & 2878 & 27.2 \\
\hline 343 & 2.42 & 8.33 & 16.5 & 3014 & 26.1 \\
\hline
\end{tabular}


to increase the absolute transmutation of ${ }^{129} \mathrm{I}$ and to enhance the LLFP discharge burnups at the same time, the above "standard" assembly designs were further optimized. As discussed in Sec. IV.A.1, the target discharge burnup increases monotonically as the moderator volume fraction increases (i.e., as the target volume fraction decreases). On the other hand, for a moderator volume fraction near that of the standard design $(60 \%)$, the absolute transmutation rates decrease as the target loading decreases. As a result, in order to increase the discharge burnup while satisfying the support ratio goal, it is necessary to increase the moderator volume while maintaining the ${ }^{99} \mathrm{Tc}$ loading and increasing the ${ }^{129} \mathrm{I}$ loading. The only possible way to accomplish this without introducing additional target assemblies is to increase the total target pin volume by reducing the coolant volume.

Accordingly, modified assembly designs were developed by reducing the pitch-to-diameter $(\mathrm{P} / \mathrm{D})$ ratio of target pins to its minimum value of 1.0, keeping the same number of target pins per assembly. In these modified designs, the target pin diameter was increased from the reference value of $0.74 \mathrm{~cm}$ to $0.89 \mathrm{~cm}$ with the same clad thickness. To maintain the ${ }^{99} \mathrm{Tc}$ loading, the moderator volume fraction within each ${ }^{99} \mathrm{Tc}$ target pin was increased from 60 to $72 \%$. Whereas, the ${ }^{129} \mathrm{I}$ loading was increased by applying a moderator volume fraction of $65 \%$; this increases the loading per assembly by $\sim 18 \%$ relative to the reference target design. Although the moderator volume fractions inside target pins are not increased much relative to the reference designs, the moderator volume fraction of the assembly is significantly enhanced, and hence the neutron spectrum is much softer in these modified designs. In the modified ${ }^{99} \mathrm{Tc}$ target assembly, the thermal neutron filter located at the core/reflector boundary was enhanced by replacing the central void of the annular target pins with HT-9; this increases the parasitic absorption of low-energy neutrons.

The resulting LLFP transmutation rates and some core performance parameters are summarized in Table XV. It can be seen that the transmutation rates obtained with the modified LLFP assembly designs are high enough to meet the support ratio goals for both ${ }^{99} \mathrm{Tc}$ and ${ }^{129} \mathrm{I}$. The modified assembly design increases the absolute and relative transmutation rates of ${ }^{129}$ I by $\sim 50$ and $\sim 10 \%$, respectively, but it increases the ${ }^{99} \mathrm{Tc}$ transmutation rates only by $\sim 1 \%$. This is attributed to moderator trends observed for the PWR LLFP transmutation analyses, which indicated that the effective capture cross section of ${ }^{129} \mathrm{I}$ is enhanced more efficiently. It is noteworthy that the total flux levels in the modified design are significantly lower than those in the standard design, due to the softened neutron spectrum. This reduced flux largely offsets the increase in capture cross sections, particularly for ${ }^{99} \mathrm{Tc}$. When the modified LLFP designs are used, the TRU inventory must be increased by $\sim 1.6 \%$ mainly because of increased ${ }^{129}$ I capture rate. The local power peaking factors obtained from the seven-assembly model VIM calculations are $<1.45$ for all cases. With appropriate coolant flow allocation, these power peaking factors can be tolerated since the power densities in the peripheral fuel assemblies are much lower than the core average power density $\left(240 \mathrm{~W} / \mathrm{cm}^{3}\right)$, as shown in Fig. 23.

The LLFP target assemblies loaded in the reflector are transmuted mainly by the neutrons leaking out of the core. As a result, the impact on the core performance parameters was generally minor. Compared to the base design without any LLFP loading, the loading of modified LLFP assemblies in the reflector region increases the TRU inventory only by $\sim 10 \%$, with a commensurate $8 \%$ reduction in the TRU discharge burnup. This increased inventory reduces the burnup reactivity swing slightly. The peak power density still occurs within the core (near the interface between inner and outer cores in Fig. 22), and the global peaking factor is basically the same as the base design.

In the modified LLFP assembly designs, the P/D ratio of target pins is 1.0, and hence the coolant flow area is reduced by a factor of $\sim 4$ relative to the ATW fuel assembly. Thus, adequate cooling of target pins must be considered. However, as shown in Fig. 23, the power densities in the LLFP target assemblies are $<10 \%$ of the core average power density $\left(240 \mathrm{~W} / \mathrm{cm}^{3}\right)$. Considering the excellent thermal properties of sodium coolant, it is expected that the LLFP target pins can be adequately cooled. However, the power density will be much increased compared to the typical steel reflector assemblies. Thus, appropriate coolant flow will need to be allocated.

\section{IMPACTS OF LONG-LIVED FISSION PRODUCT TRANSMUTATION ON YUCCA MOUNTAIN REPOSITORY}

Current repository performance evaluations of the Yucca Mountain repository show ${ }^{237} \mathrm{~Np}$ as the largest

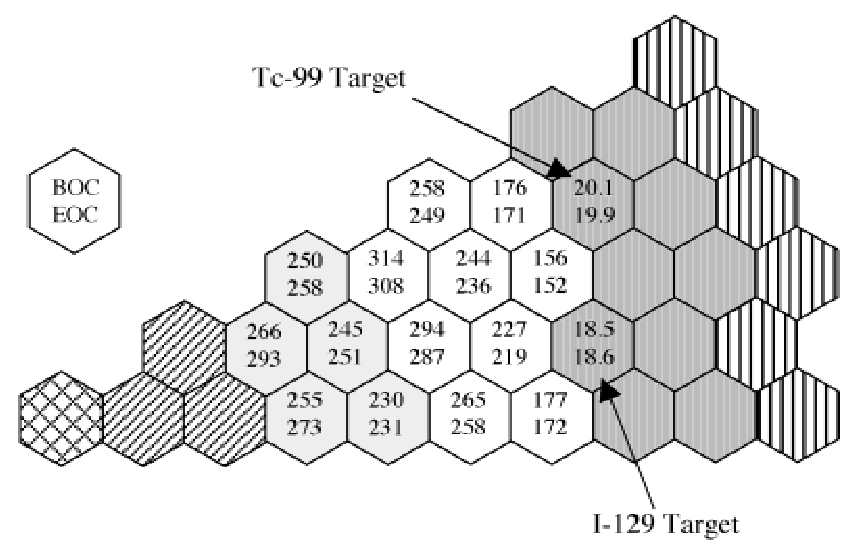

Fig. 23. Assembly average power densities (watts per cubic centimeter) of equilibrium cycle ATW with ${ }^{99} \mathrm{Tc} /{ }^{129} \mathrm{I}$ target assemblies $(\mathrm{P} / \mathrm{D}=1.0)$ in the reflector region. 
TABLE XV

Transmutation Performance Parameters of Simultaneous Loading of ${ }^{99} \mathrm{Tc}$ and ${ }^{129} \mathrm{I}$ in Reflector Region of Equilibrium Cycle ATW Core

\begin{tabular}{|c|c|c|}
\hline \multirow[b]{2}{*}{ Parameter } & \multicolumn{2}{|c|}{ LLFP Assembly } \\
\hline & $\begin{array}{c}\text { Standard } \\
(\mathrm{P} / \mathrm{D}=1.1969)\end{array}$ & $\mathrm{P} / \mathrm{D}=1.0$ \\
\hline $\begin{array}{l}\text { LLFP transmutation rate over } 14 \text { cycles (1890 EFPDs) } \\
\text { BOEC a inventory }(\mathrm{kg}) \\
{ }^{99} \mathrm{Tc} \\
{ }^{129} \mathrm{I} \\
\text { Average } \% / \text { cycle } \\
{ }_{99} \mathrm{Tc} \\
{ }^{129} \mathrm{I} \\
\text { Average kg/cycle } \\
{ }^{99} \mathrm{Tc} \\
{ }^{129} \mathrm{I} \\
\text { Discharge burnup }(\text { at. } \%) \\
{ }^{99} \mathrm{Tc} \\
{ }^{129} \mathrm{I}\end{array}$ & $\begin{array}{c}531.1 \\
69.2 \\
\\
2.08 \\
2.40 \\
\\
12.9 \\
2.00 \\
\\
29.1 \\
33.7\end{array}$ & $\begin{array}{r}530.1 \\
91.5 \\
\\
2.10 \\
2.65 \\
\\
13.0 \\
2.99 \\
\\
29.4 \\
37.1\end{array}$ \\
\hline $\begin{array}{l}\text { TRU fuel } \\
\text { BOEC }{ }^{\text {a }} \text { inventory }(\mathrm{kg}) \\
\text { Discharge burnup (at.\%) }\end{array}$ & $\begin{array}{l}2980 \\
26.4\end{array}$ & $\begin{array}{l}3027 \\
26.0\end{array}$ \\
\hline $\begin{array}{l}\text { Local power peaking in seven-assembly model } \\
{ }_{99} \mathrm{Tc} \text { target } \\
{ }^{129} \mathrm{I} \text { target }\end{array}$ & $\begin{array}{l}1.43 \\
1.40\end{array}$ & $\begin{array}{l}1.45 \\
1.44\left(1.88^{\mathrm{b}}\right)\end{array}$ \\
\hline $\begin{array}{l}\text { Initial total flux in LLFP assembly }\left(\mathrm{n} / \mathrm{cm}^{2} \cdot \mathrm{s}\right) \\
{ }^{99} \mathrm{Tc} \text { target } \\
{ }^{129} \text { I target }\end{array}$ & $\begin{array}{l}9.28 \mathrm{E}+14^{\mathrm{c}} \\
1.35 \mathrm{E}+15\end{array}$ & $\begin{array}{l}7.02 \mathrm{E}+14 \\
1.09 \mathrm{E}+15\end{array}$ \\
\hline $\begin{array}{l}\text { Peak discharge fast fluence in LLFP assembly }\left(\mathrm{n} / \mathrm{cm}^{2}\right) \\
{ }^{99} \mathrm{Tc} \text { target } \\
{ }^{129} \mathrm{I} \text { target }\end{array}$ & $\begin{array}{l}1.77 \mathrm{E}+23 \\
2.40 \mathrm{E}+23\end{array}$ & $\begin{array}{l}1.65 E+23 \\
2.25 E+23\end{array}$ \\
\hline
\end{tabular}

${ }^{\text {a} B O E C ~}=$ beginning-of-equilibrium cycle.

${ }^{\mathrm{b}}$ HT-9 rods are used instead of ${ }^{99} \mathrm{Tc}$ rod as the thermal neutron filter.

${ }^{\mathrm{c}}$ Read as $9.28 \times 10^{14}$.

contributor to the long-term dose release rate with ${ }^{99} \mathrm{Tc}$ and ${ }^{129}$ I the major LLFP contributors. In addition, previous studies reveal that removal of $99.9 \%$ actinides from the waste might reduce the long-term (beyond $100000 \mathrm{yr}$ ) peak dose rate by a factor of 156 (Ref. 22). It was found that ${ }^{99} \mathrm{Tc}$ was the dominant contributor to the dose risk when $99.9 \%$ actinides were simply removed without taking into account the conversion of the actinides to FPs. Based on this study, the impacts of LLFP transmutation on the Yucca mountain repository were analyzed by comparing three cases:

1. direct disposal of spent fuel

2. transmutation of TRU into FPs

3. transmutation of both TRU and LLFP.
The additional FP inventory generated by actinide transmutation depends on the specific transmutation scenario and method. However, to estimate the impact, it was assumed that among $99.9 \%$ actinides removed from the spent fuel, all TRUs were transmuted into FPs, and the uranium elements were stored for future utilization. The production rates of ${ }^{99} \mathrm{Tc}$ and ${ }^{129} \mathrm{I}$ from actinide transmutation were approximated by their weight fractions among the total FPs in the typical PWR spent fuel with $33 \mathrm{GWd} /$ tonne U burnup and 25 -yr cooling. That is, it was assumed that 2.3 and $0.52 \%$ of TRU were converted to ${ }^{99} \mathrm{Tc}$ and ${ }^{129} \mathrm{I}$, respectively.

When the ${ }^{99} \mathrm{Tc}$ and ${ }^{129} \mathrm{I}$ are recycled and transmuted into short-lived isotopes, the cumulative fractional loss to the repository was estimated by summing the initial spent-fuel processing loss and the cumulative loss 
TABLE XVI

Inventories of ${ }^{99} \mathrm{Tc}$ and ${ }^{129} \mathrm{I}$ to Be Sent to the Repository*

\begin{tabular}{|c|c|c|c|c|c|c|}
\hline \multirow[b]{3}{*}{ Isotope } & \multirow[b]{3}{*}{$\begin{array}{c}\text { Direct } \\
\text { Disposal }\end{array}$} & \multirow{3}{*}{$\begin{array}{c}\text { TRU } \\
\text { Transmutation } \\
\text { (No LLFP } \\
\text { Transmutation) }\end{array}$} & \multicolumn{4}{|c|}{ TRU and LLFP Transmutation } \\
\hline & & & \multicolumn{2}{|c|}{$5 \%$ LLFP Loss Factor } & \multicolumn{2}{|c|}{$1 \%$ LLFP Loss Factor } \\
\hline & & & $\begin{array}{c}\text { Low } \\
\text { Burnup }^{a}\end{array}$ & $\begin{array}{c}\text { High } \\
\text { Burnup }^{\mathrm{b}}\end{array}$ & $\begin{array}{c}\text { Low } \\
\text { Burnup }^{a}\end{array}$ & $\begin{array}{c}\text { High } \\
\text { Burnup }^{b}\end{array}$ \\
\hline $\begin{array}{l}{ }^{99} \mathrm{Tc} \\
{ }^{129} \mathrm{I}\end{array}$ & $\begin{array}{l}6.37 \mathrm{E}+7^{c} \\
1.45 \mathrm{E}+7\end{array}$ & $\begin{array}{l}8.04 \mathrm{E}+7 \\
1.82 \mathrm{E}+7\end{array}$ & $\begin{array}{l}1.77 \mathrm{E}+7 \\
6.37 \mathrm{E}+6\end{array}$ & $\begin{array}{l}1.20 \mathrm{E}+7 \\
2.72 \mathrm{E}+6\end{array}$ & $\begin{array}{l}4.02 \mathrm{E}+6 \\
1.64 \mathrm{E}+6\end{array}$ & $\begin{array}{l}2.62 \mathrm{E}+6 \\
5.93 \mathrm{E}+5\end{array}$ \\
\hline
\end{tabular}

*The inventories are given in grams.

${ }^{\mathrm{a}} 19$ at. $\%$ for ${ }^{99} \mathrm{Tc}$ and 10 at. $\%$ for ${ }^{129} \mathrm{I}$.

b30 at.\% for both ${ }^{99} \mathrm{Tc}$ and ${ }^{129} \mathrm{I}$.

${ }^{\mathrm{c}}$ Read as $6.37 \times 10^{7}$.

resulting from the recycling of LLFP targets. Denoting the fractional discharge burnup of LLFP and the fraction of LLFP lost in recycle/refabrication by $B$ and $l$, respectively, the cumulative fractional loss $L_{T}$ can be approximately represented as

$$
L_{T}=\frac{l}{B+(1-B) l} .
$$

Under these assumptions, the ${ }^{99} \mathrm{Tc}$ and ${ }^{129} \mathrm{I}$ inventories sent to the repository were evaluated for the three specific cases. The initial inventories were obtained from previous Yucca Mountain performance assessment studies. The total initial inventory consists of 7860 commercial spent nuclear fuel packages from PWRs and boiling water reactors and 3890 codisposal packages for U.S. Department of Energy spent fuel and high-level waste glass. The inventories for the recycled LLFP case were estimated using two reprocessing loss fractions and two sets of discharge burnups. As the reprocessing loss fraction, a recommended value of $5 \%$ and a low loss of $1 \%$ were used. In the first set of discharge burnups, the LLFP discharge burnups in PWRs given in Sec. III were used, i.e., 19 and 10 at.\% for ${ }^{99} \mathrm{Tc}$ and ${ }^{129} \mathrm{I}$ discharge burnups, respectively. In the second set, both discharge burnups were assumed to be 30 at.\%, which could be achieved by using a special moderated LLFP target assembly in the fast ATW system. Table XVI summarizes the resulting ${ }^{99} \mathrm{Tc}$ and ${ }^{129} \mathrm{I}$ inventories to be sent to the repository.

The impact of ${ }^{99} \mathrm{Tc}$ and ${ }^{129} \mathrm{I}$ transmutation on the repository performance was estimated by evaluating the dose rate resulting from use of water from a well located $\sim 20 \mathrm{~km}$ from the repository, which is one of the key parameters considered in the total system performance assessment for the site recommendation of the Yucca mountain repository. Since ${ }^{99} \mathrm{Tc}$ and ${ }^{129} \mathrm{I}$ are highly soluble in water, the release rates of these isotopes from the engineered barrier system (EBS) are proportional to their inventories. Furthermore, since they are nonsorbing elements, the temporal shapes and magnitudes of the EBS release rates and the release rate at the $20-\mathrm{km}$ well are very similar with a time delay of only one or two thousand years. Therefore, the dose rates of ${ }^{99} \mathrm{Tc}$ and ${ }^{129} \mathrm{I}$ for different inventories were estimated in a simple way by linearly combining the temporal shapes of individual isotopes provided from previous repository analyses. ${ }^{22}$

In Fig. 24, both total dose rate and major dose contributors are reproduced for the direct disposal case. It can be seen that the long-term dose rate is dominated by ${ }^{237} \mathrm{~Np}$, and the dose contributions of ${ }^{99} \mathrm{Tc}$ and ${ }^{129} \mathrm{I}$ are marginal, although ${ }^{99} \mathrm{Tc}$ is the major dose contributor during the early time period. Figure 25 shows that ${ }^{99} \mathrm{Tc}$ becomes the dominant contributor to the dose rate if 99.9\% of actinides are removed from the spent fuel and all the removed TRUs are transmuted into FPs. In this

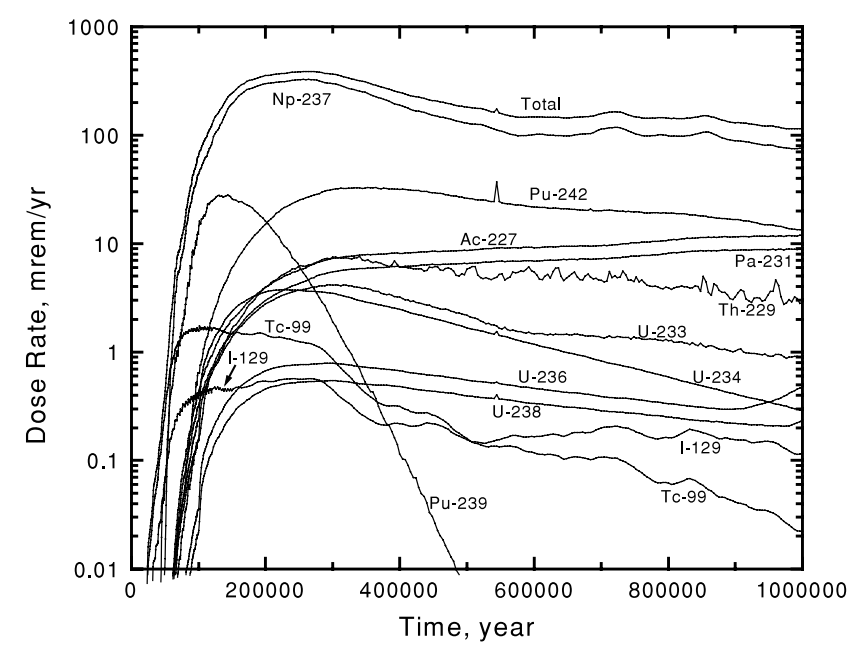

Fig. 24. Estimated dose rates at the 20-km well of the direct disposal case. 


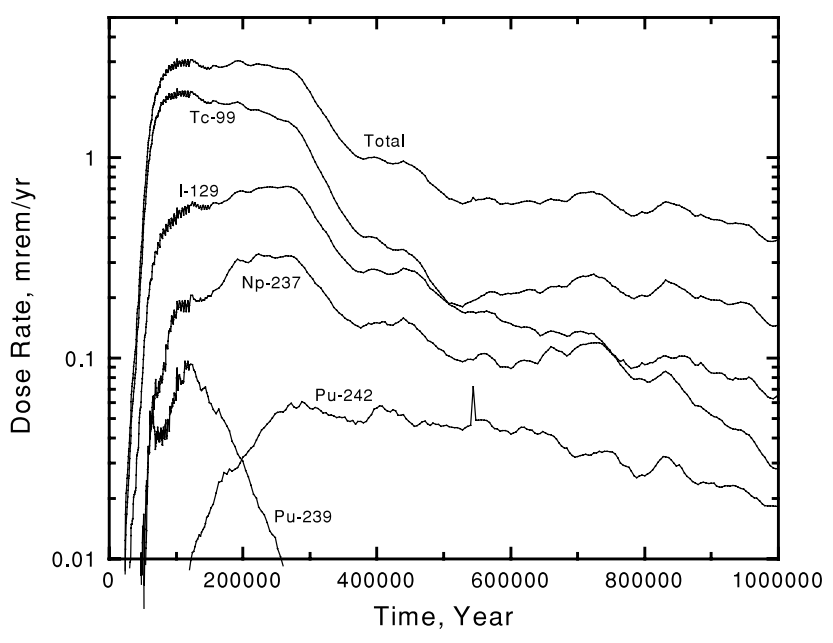

Fig. 25. Estimated dose rates at the $20-\mathrm{km}$ well of the TRU transmutation case.

case, the peak dose rate is $\sim 3 \mathrm{mrem} / \mathrm{yr}$, which is only $\sim 20 \%$ higher than the simple removal of $99.9 \%$ actinides. Figure 26 shows the impact on the dose rate of the base ${ }^{99} \mathrm{Tc} /{ }^{129} \mathrm{I}$ transmutation scenario (discharge burnups of ${ }^{99} \mathrm{Tc}$ and ${ }^{129} \mathrm{I}$ are 19 and 10 at.\%, respectively, with recycle loss factor of 5\%). In this case, the ${ }^{99} \mathrm{Tc}$ and ${ }^{129} \mathrm{I}$ transmutation reduces the peak dose rate from $\sim 3$ to $\sim 1 \mathrm{mrem} / \mathrm{yr}$. The factor of $\sim 3$ reduction in LLFP dose rate could also be achieved using special tailored waste forms. In fact, previous Argonne National Laboratory studies on the metallic waste form (where Tc naturally resides for pyroprocessing) indicated superior leach resistance compared to typical glass waste forms. It can be deduced from the inventories presented in Table XVI

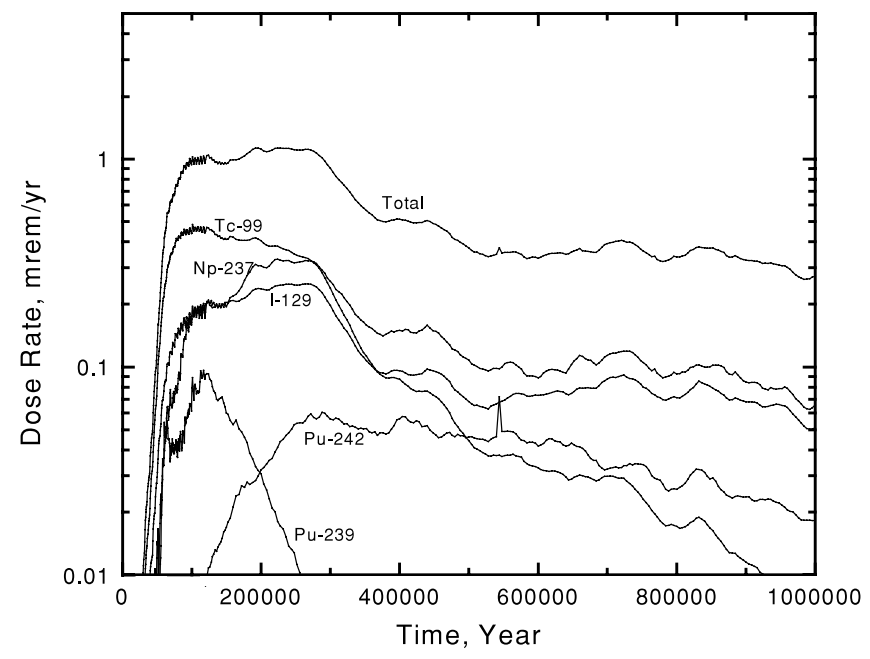

Fig. 26. Estimated dose rates at the $20-\mathrm{km}$ well of the TRU and LLFP transmutation case. that a higher LLFP discharge burnup and a lower reprocessing loss fraction would further reduce the dose rate of the LLFPs significantly in terms of relative value.

Given a repository dose limit of $15 \mathrm{mrem} / \mathrm{yr}$, the current Yucca Mountain release evaluations do not indicate a compelling need to transmute ${ }^{99} \mathrm{Tc}$ and ${ }^{129} \mathrm{I}$, where the maximum doses rates are only 1 to $3 \mathrm{mrem} / \mathrm{yr}$. However, it is important to note that the LLFPs are much more mobile than the TRU species, and the dose rates for the first $40000 \mathrm{yr}$ following closure are completely dominated by the LLFPs, and ${ }^{99} \mathrm{Tc}$ in particular. The current repository design relies on robust, long-lived waste containers that do not even begin to fail until the 10000-yr time frame. This allows retention of the bulk of the ${ }^{99} \mathrm{Tc}$ inventory until its elimination by decay (10000-yr halflife) becomes important. Furthermore, the ${ }^{99} \mathrm{Tc}$ release and transport is not limited by solubility; the "short term" ${ }^{99} \mathrm{Tc}$ dose rates are directly tied to the container and waste form failure rates. Therefore, elimination of the LLFP inventory could allow significant relaxation of the waste form and container performance criteria, with associated economic benefits. In addition, future utilization of the remaining uranium inventories could result in large increases in the LLFP waste inventory; thus, some development of either specialized waste form or transmutation target is prudent.

\section{CONCLUSIONS}

A systematic study on the transmutation of LLFPs has been performed with the aim of devising an optimal strategy for their transmutation in critical or subcritical reactor systems and evaluating impacts on the geologic repository. First, ${ }^{99} \mathrm{Tc}$ and ${ }^{129} \mathrm{I}$ were confirmed to have highest transmutation priorities in terms of transmutability and long-term radiological risk reduction. Then, the transmutation potentials of thermal and fast systems for ${ }^{99} \mathrm{Tc}$ and ${ }^{129} \mathrm{I}$ were evaluated by considering a typical PWR core and a sodium-cooled ATW system. To determine the best transmutation capabilities, various target design and loading optimization studies were performed. Finally, based on these results, the impacts of ${ }^{99} \mathrm{Tc}$ and ${ }^{129}$ I transmutation on the Yucca mountain repository were assessed in terms of the dose rate.

For ${ }^{99} \mathrm{Tc}$ transmutation in a PWR core, thin ${ }^{99} \mathrm{Tc}$ film coating of fuel pellet, homogeneous mixing of ${ }^{99} \mathrm{Tc}$ with fuel, and mixture of ${ }^{99} \mathrm{Tc}$ with $\mathrm{ZrH}_{2}$ are preferable loading options from the incineration point of view. However, the Tc-bearing fuel options need to be evaluated carefully in terms of fuel fabrication, performance, and $\mathrm{Tc}$ recovery rate. In the case of ${ }^{129} \mathrm{I}$ targets loaded in the guide-tube locations, annular iodine targets filled with $\mathrm{ZrH}_{2}$ moderator have the best transmutation performance. However, considering the xenon gas pressure buildup, an annular target with inner void zone would be preferable to other options. 
Both ${ }^{99} \mathrm{Tc}$ and ${ }^{129} \mathrm{I}$ can be stabilized in the same PWR core at the cost of higher fuel enrichment. However, due to the enrichment constraint, it appears that only one of them can be incinerated in a given burner core with a support ratio of $\sim 0.5$ for ${ }^{99} \mathrm{Tc}$ and $\sim 3$ for ${ }^{129} \mathrm{I}$. In a $\mathrm{UO}_{2}$ core, the discharge burnups of ${ }^{99} \mathrm{Tc}$ and ${ }^{129}$ I are $\sim 20$ and $\sim 12 \%$, respectively, for an 18 -month fuel cycle with an $80 \%$ capacity factor. For a MOX core, the LLFP discharge burnup is $\sim 19 \%$ smaller for ${ }^{99} \mathrm{Tc}$ and $\sim 50 \%$ smaller for ${ }^{129} \mathrm{I}$, relative to the $\mathrm{UO}_{2}$ fuel case. The safety-related reactivity coefficients are not affected significantly by the LLFP loading, if the LLFP loading is restricted by the fuel enrichment constraint $(5.0 \mathrm{wt} \%$ $\left.{ }^{235} \mathrm{U}\right)$.

In the sodium-cooled ATW system, the ${ }^{99} \mathrm{Tc}$ and ${ }^{129} \mathrm{I}$ transmutation rates are significantly enhanced by employing $\mathrm{ZrH}_{2}$ moderator. The annular target configuration filled with the moderator reduces the self-shielding effects and increases the effective capture cross sections relative to the distinct target and moderator pin configuration. There exists an optimal moderator volume fraction to maximize the absolute transmutation rate. However, the fractional LLFP transmutation rate increases monotonically with the moderator volume fraction (i.e., as the target loading decreases). For a fixed LLFP loading, the moderator volume needs to be maximized to increase the transmutation rate. The local power peaking in the neighboring fuel assemblies can be effectively mitigated by employing unmoderated ${ }^{99} \mathrm{Tc}$ target pins at the interface between fuel and target assemblies.

The heterogeneous loading of LLFP target material in special moderated assemblies is more favorable than the homogeneous mixing of target and fuel in terms of the relative transmutation rate. Among various heterogeneous loading options, the loading in the reflector region seems to be the best option from the neutron economy and safety point of view. This option provides sufficient transmutation rates to meet the LLFP support ratio goal to balance TRU transmutation of the ATW system. By a simultaneous loading of twelve ${ }^{99} \mathrm{Tc}$ and twelve ${ }^{129} \mathrm{I}$ target assemblies in the reflector region, ${ }^{99} \mathrm{Tc}$ and ${ }^{129} \mathrm{I}$ can be consumed with a support ratio equal to the TRU support ratio $(\sim 3.2)$ at the cost of increased fuel inventory (and thus decreased discharge burnup) by $\sim 10 \%$. Discharge burnups of $\sim 29$ and $\sim 37 \%$ are achieved for ${ }^{99} \mathrm{Tc}$ and ${ }^{129}$ I target assemblies with an $\sim 5$-yr irradiation period. The discharge burnup could be further increased by increasing the number of target assemblies with the correspondingly reduced loading per assembly.

The current Yucca Mountain release evaluations do not indicate a compelling need to transmute ${ }^{99} \mathrm{Tc}$ and ${ }^{129} \mathrm{I}$ because the dose rates are well below current regulatory limits for the spent-fuel LLFP inventory. However, elimination of the LLFP inventory could allow some relaxation of the stringent waste form and container performance criteria, with associated economic benefits. Therefore, some development of either specialized waste forms or transmutation target for the LLFP is prudent, especially when sustained fission power production (with accumulating FP inventory) is envisioned for future applications.

\section{ACKNOWLEDGMENT}

The authors are grateful to E. E. Morris for providing the isotopic dose rates of the Yucca mountain repository.

\section{REFERENCES}

1. "A Roadmap for Developing Accelerator Transmutation of Waste (ATW) Technology," DOE/RW-0519, U.S. Department of Energy (1999).

2. W. S. YANG and H. S. KHALIL, "Blanket Design Studies of a Lead-Bismuth Eutectic-Cooled Accelerator Transmutation of Waste System,” Nucl. Technol., 135, 162 (2001).

3. J. L. KLOOSTERMAN and J. M. LI, "Transmutation of Tc-99 in Fission Reactors," Proc. 3rd Int. Information Exchange Mtg. Actinide and Fission Product Partitioning and Transmutation, Cadarache, France, NEA/P\&T Report 13, p. 285, Organisation for Economic Co-operation and Development (1995).

4. K. D. DOBBIN, D. P. JORDHEIM, D. W. WOOTAN, and J. A. RAWLINS, "Transmutation of LWR High-Level Waste in LMRs," Trans. Am. Nucl. Soc, 64, 122 (1991).

5. T. WAKABAYASHI and N. HIGANO, "Study on MA and FP Transmutation in Fast Reactors," Prog. Nucl. Energy, 32, 555 (1998).

6. H. GOLFIER et al., "Parametrical Analysis of Tc-99 and I-129 Transmutation in Reactor," Proc. Int. Conf. Future Nuclear Systems (GLOBAL '99), Jackson Hole, Wyoming, August 29-September 3, 1999 (1999).

7. W. S. PARK, Y. H. KIM, C. K. PARK, J. S. CHUNG, and C. H. KIM, "A Design Study of Tc-99 and I-129 Transmutation in the HYPER System," Nucl. Sci. Eng. 143, 188 (2002).

8. D. W. WOOTAN, D. P. JORDHEIM, and W. Y. MATSUMOTO, "Irradiation Test of Tc-99 and I-129 Transmutation in the Fast Flux Test Facility," Trans. Am. Nucl. Soc., 64, 125 (1991).

9. R. J. M. KONINGS, W. M. P. FRANKEN, R. P. CONRAD, J.-F. GUEUGON, and J.-C. SPIRLET, "Transmutation of Technetium and Iodine-Irradiation Tests in the Frame of the EFTTRA Cooperation,” Nucl. Technol., 117, 293 (1997).

10. A. CONTI et al., "Long-Lived Fission Product Transmutation Studies," Proc. Int. Conf. Future Nuclear Systems (GLOBAL '99), Jackson Hole, Wyoming, August 29-September 3, 1999 (1999). 
11. R. N. HILL et al., "Physics Studies of a Sodium Cooled ATW Design," Proc. Technical Committee Mtg. Core Physics and Engineering Aspects of Emerging Nuclear Energy Systems for Energy Generation and Transmutation, Argonne, Illinois, November 28-December 1, 2000, International Atomic Energy Agency (2000).

12. "Viability Assessment of a Repository at Yucca Mountain," DOE/RW-0508, U.S. Department of Energy (1998).

13. A. G. CROFF, "A User's Manual for the ORIGEN2 Computer Code," ORNL/TM-7175, Oak Ridge National Laboratory (1980).

14. T. K. KIM, J. A. STILLMAN, and T. A. TAIWO, "Assessment of TRU Stabilization in PWRs," ANL-AAA-020, Argonne National Laboratory (2002).

15. "WIMS - A Modular Scheme for Neutronics Calculations, User's Guide for Version 8," ANSWERS/WIMS(99)9, The ANSWERS Software Package, AEA Technology.

16. P. BARBRAULT, "A Plutonium-Fueled High-Moderated Pressurized Water Reactor for the Next Century," Nucl. Sci. Eng., 122, 240 (1996).
17. M. SALVATORES, I. SLESSAREV, and TCHISTIAKOV, "The Transmutation of Long-Lived Fission Products by Neutron Irradiation," Nucl. Sci. Eng., 130, 309 (1998).

18. J. E. LAIDLER et al., "A Roadmap for Developing ATW Technology: Separation \& Waste Forms Technology," ANL99/15, Argonne National Laboratory (1999).

19. R. N. BLOMQUIST, "VIM-A Continuous Energy Monte Carlo Code at ANL," Proc. Seminar-Workshop Review of the Theory and Application of Monte Carlo Methods, April 21-23, 1980, ORNL/RSIC-44, Oak Ridge National Laboratory (1980).

20. B. J. TOPPEL, "A User's Guide to the REBUS-3 Fuel Cycle Analysis Capability," ANL-83-2, Argonne National Laboratory (1983).

21. H. HENRYSON II, B. J. TOPPEL, and C. G. STENBERG, "MC 2 -2: A Code to Calculate Fast Neutron Spectra and Multigroup Cross Sections," ANL-8144, Argonne National Laboratory (1976).

22. E. E. MORRIS et al., "Impacts of Actinide Removal on Waste Disposal in a Geological Repository," Proc. 5th Topl. Mtg. DOE Spent Nuclear Fuel and Fissile Material Management, Charleston, South Carolina, September 16-19, 2002, American Nuclear Society (2002) (CD-ROM). 